\title{
AN EFFECTIVE NONLOCAL MEANS IMAGE DENOISING FRAMEWORK BASED ON NON-SUBSAMPLED SHEARLET TRANSFORM
}

\author{
${ }^{1}$ Bhawna Goyal, ${ }^{2}$ Ayush Dogra, ${ }^{3}$ Arun Kumar Sangaiah \\ ${ }^{1}$ Department of ECE, Chandigarh University, Mohali, India \\ ${ }^{2}$ Ronin Institute, Montclair, NJ 07043 USA \\ ${ }^{3,4}$ School of Computing Science and Engineering, Vellore Institute of Technology, Vellore, India \\ ${ }^{4}$ Department of Industrial Engineering and Management, National Yunlin University of Science and Technology, \\ Taiwan
}

\begin{abstract}
Image denoising is a fundamental task in computer vision and image processing system with an aim of estimating the original image by eliminating the noise and artifact from the noise-corrupted version of the image. In this study, a nonlocal means (NLM) algorithm with NSST (non-subsampled shearlet transform) has been designed to surface a computationally simple image denoising algorithm. There are three steps in our process; First, NSST is employed to decompose source image into coarser and finer layers. The number of decomposition level of NSST is set to two, resulting in one low frequency coefficient (coarser layer) and four high frequency coefficients (finer layers). The two levels of decomposition are used in order to preserve memory, reduce processing time, and reduce the influence of noise and misregistration errors. The finer layers are then processed using NLM algorithm, while the coarser layer is left as it is. The NL-Means algorithm reduces noise in finer layers while maintaining the sharpness of strong edges, such as the image silhouette. When compared to noisy images, this filter also smoothes textured regions, resulting in retaining more information. To obtain a final denoised image, inverse NSST is performed to the coarser layer and the NL-means filtered finer layers. The robustness of our method has been tested on the different multisensor and medical image dataset with diverse levels of noise. In the context of both subjective assessment and objective measurement, our method outperforms numerous other existing denoising algorithms notably in terms of retaining fine image structures. It is also clearly exhibited that the proposed method is computationally more effective as compared to other prevailing algorithms.
\end{abstract}

Keywords: non-local means, image denoising, multiscale decomposition, nonsubsampled Laplacian pyramid (NSLP), shearlet filter

\section{INTRODUCTION}

Image denoising is the process to eliminate noise or distortions from the images. It is mostly used as image preor post-processing to boost the quality of the processed images for further image analysis and understanding. Digital image is often prone to noise degradation during the image acquisition in imaging systems owing to sensor characteristics and complex camera processing procedures [1-2]. The elimination of noise from the captured image is a necessary step for improving image quality in computer vision applications [3-4]. Denoising of images in general seeks to obtain a clear image $x$ from a noisy observation $y=x+n$, in which $n$ is the corrupted noise. An AWGN (additive white Gaussian noise) having standard deviation $(\sigma)$ is a popularly used assumption on $n$. In particular, in order to remove noise that is varying in different settings and follows normal distribution [5], has recently got a lot of attention. Image priors are of key importance for image denoising from the Bayesian perspective [6]. In the past few decades, several techniques have been proposed to exploit the image priors for image denoising [7-8], and other image reconstruction works [9]. These algorithms are roughly categorised into nonlocal self-similarity (NSS) related algorithms [10], low rankness or sparsity related methods [13], dictionary learning related methods [11], generative learning related algorithms [12], and discriminative learning related algorithms [13] are few examples. The NSS prior is derived from the fact that a local patch in the natural image has several nonlocal similar patches across the images where, Euclidean distance is often used to measure the similarity. Existing denoising algorithms have successfully utilized the NSS prior for example BM3D [14], WNNM [15], and N3Net [16], among others. Regardless their ability to improve denoising quality, this patch level NSS prior utilized in this method undergo one major obstacle i.e. they tend to introduce artefacts around the edges. This can be attributed to the fact that finding nearly identical patches for complete reference patches in the natural image is quite difficult, particularly when the number of identical patches is more. BM3D-SAPCA [17] 
proposed a solution to overcome this flaw of finding shape adaptive similar patches. Further another improvement can be observed in [18]. However, shape artefacts would be included in the denoised images as a result. Multiscale strategies [19] has been introduced for improving the similarity, however the information would be lost in the course level and same counterparts would be failed to detect. In this work, we take the advantage of both spatial and transform domain denoising by integrating the nonlocal means algorithm and NSST. The key concept of our work is demonstrated in Figure 1. With the help of multiscale directional transform, i.e. the geometry of multidimensional data can be captured using the NSST. Shear parameters is then used to capture the singularities. The non-local mean filtering facilitates preservation of edge features and details of the multi-resolution representation. High-contrast elements such as textures in general are rarely preserved while suppressing noise. As a result, this serves as a motivation to use a combination of non-subsampled Shearlet transform and a nonlocal means algorithm to denoise images. The advantages of combined adaptable approach especially exhibit the capacity to extract multidimensional data geometry. It could also effectively indicate edges in high-noise images.

The remainder of this paper is organised as follows: Section 2 broadly summarise the previous related works. Section 3 illustrated a key concept of NSST. The proposed image denoising method using NSST decomposition and the non-local means algorithm is demonstrated in Section 4. Section 5 has been dedicated to exhibit the numerous experimental results which demonstrates the analysis, discussions, and efficiency of our method with other comparative methods. Finally, a summary of the conclusion is mentioned in Section 6.

\section{SUMMARY OF PREVIOUS WORKS}

In this section, recent developments of image denoising are presented and discussed. Self-similar patches are used in many important denoising algorithms; NLM (Non-Local Means Algorithm) [20] and BM3D (Block Matching 3D transform) [21]. Many versions have been proposed as a result of their improvisation and evolution, including SADCT (Shape Adaptive Discrete Cosine Transform) [22], SAPCA (Shape Adaptive Principal Component Analysis) [17] and many more that explore self-similar patches in transform domain. The dictionary learning oriented algorithms [23] use self-identical patches and learn overcomplete dictionaries from clear image to recognise and formulate sparsity. Numerous algorithms [24-25] have looked for using the maximum likelihood approach to train the statistical priors, such as the gaussian mixture model (GMM) of natural patches or patch reconstruction. For multi-resolution study on transform domain methods, a novel multiscale directional transform known as shearlet has been proposed in literature. The single Shearlet function [26] is defined by the direction of the singularities, a translation and a shear. However, it has unbound support depending on the space domain, as well as functions that are band-limited. As a result, the spatial domain efficiently helps the compact representation components for local characteristics of any images. [27]. NSST can also accurately characterise an image's geometric and textural features [28]. It has the ability to breakdown an image into the number of directional elements. A NSST has a lower computing complexity and sparse approximation capabilities than the NSCT (nonsubsampled contourlet transform) [29]. By introducing the texture measure as smooth penalty weight or spatially varying data fidelity in the sparse norm \& non local total variation algorithms, the nonlocal version of generalised RTV (NLGRTV) for denoising has been introduced in [30]. The important concept is to employ the updated texture measure as the spatially changing penalty weight $\&$ substitute local candidate pixel in the smoothpenalty term with the nonlocal set [30]. Takeda et al. [31] extend the application of kernel regression to deblurring. This algorithm employed a novel image prior that generalises some of the most widely used regularisation techniques. Chambolle et al. [32] addressed a wide range of theoretical and practical aspects of Total Variationbased image reconstruction algorithms. Graham Treece has suggested a rather recent filter based on morphological filtering for adaptively eliminate the quantity of noise contain in the images. It is called bitonic filtering and works on the idea of bitonicity which means it keeps image information that is locally bitonic i.e. a signal comprising of one minima or maxima in the given range. It targets to remove the noise pixels that are constantly varying with low range of frequency. The bitonic filtering is the nonlocal filter that creates a weighted gaussian result by combining the opening \& closing weights of filter. It is a type of adaptive image denoising that keeps edges whilst reducing noise without requiring advance knowledges of the quantity of noise. In case of AWGN and impulse noise, this filter has superior denoising efficiency than median, gaussian etc filters [33]. B.K Shreyamsha presented a noise thresholding and Gaussian/Bilateral filtering-based denoising method. An idea of method noise has been proposed in this scheme. The dissimilarity between the input image and denoised image using a particular approach is referred to as method noise. The noisy images are supposed to be contaminated/degraded via Gaussian Additive noise having zero mean \& a known variance has been regulated to test at low and high noise value in GBFMT [34]. The contaminated images are filtered utilizing bilateral filtering \& the resulting residual images are hard thresholded in the wavelet domain. In a similar study, a non-local filter was used instead of a bilateral filter to take use of the idea of method noise thresholding (NLMNT) [35]. A method known as WBF (weighted bilateral filter) has been developed optimising standard bilateral filtering and its adaptation in the weighted form with the goal of minimising the MSE (man square error). Chaudary et al. presented the sure \& fast strategy for image 
denoising by utilising bilateral filter [36]. Random field methods are another popular topic that is often used in image denoising along with other low-level processing applications including image segmentation \& classification. In this algorithm, the intensity of a pixel is determined by its neighbours. These algorithms are primarily based on the principle that the global representation of images may be generated through its local physical structure, that is done using the conditional probability distribution function known as markov random field (MRF) [37]. Tomasi and Munich proposed the bilateral filter (BF) as the improvised version of neighbouring filter that weighs the distance to the reference pixels rather than following the fixed neighbourhood [38]. The authors of the paper [39] have summarised the comparison classification and assessment of several image denoising algorithms. A large number of researchers have put in a lot of time \& efforts to create a structural literature that shows significant progressive growth achieved through the series of sequential incremental enhancements. Goyal et al. [40] proposed an effectual denoising method based on NSST domain morphological filtering and Bitonic filtering. With the use of morphological techniques structural information and contrast has been regulated. NSST accurately represents the detailed directional features [41]. In multi-baseline InSAR (interferometric synthetic aperture radar), interferometric phase filtering is a critical step. Multi-baseline interferometric phase filtering methods primarily follow single baseline INSAR approaches and which do not fully exploit its data supremacy is being proposed in [42] i.e. statistically-based joint filtering of multi-baseline InSAR. An innovative framework for denoising of images based on NSST and bilateral filtering is discussed in [43]. This method employs NSST to separate high and low frequency coefficients of a noisy input image. The noise from the low frequency coefficient is removed using the weighted bilateral filter (WBF), while noise from the high frequency coefficient is removed using thresholding [43]. The continued advancement and widespread uses of CT (computed tomography) in medical imaging has increased the exposure of high radiation doses to the patients. However, using low radiation dose might result in increased noise and artefacts that adversely affects radio-diagnosis. This issue has been addressed in the nonsubsampled shearlet (NSST) domain in [44]. It is a strategy based on a novel shrinkage function. The proposed approach uses SURE-LET (stein unbiased risk of estimation \& liner expansion of threshold) technique for effectively modelling noise on multivariate shrinkage algorithms. The enhanced nonlocal means (NLM) along with NSST been used to develop a new denoising methodology for MRI images [45]. The parameters have been tuned to maximise output while maintaining highquality denoising. The image restoration algorithms have largely focussed on removal of AWGN as it degrades the uniform information subsequent and hinders subsequent image processing at large. A new framework for multi-level image denoising is proposed in [46] which progressively reduces Gaussian noise while retaining information as much as possible. The advantages of complex valued process such as the closeness of the convolution provided by the tensor products of $1 \mathrm{D}$ complex valued filters, noise stability of residual blocks and nonlinear activation on are exploited by introducing a CNN for denoising of images with key computational operation described in the complex number field in [47]. A novel model based denoising approach has been proposed by incorporating the nonlinear filtering operator, a reliability matrix, and a high-dimensional feature transformation function into the traditional consistency prior to simultaneously incorporate the valuable achievements of traditional methods into the network design while also improving network interpretability [48].

\section{NONSUBSAMPLED SHEARLET TRANSFOMR [NSST]}

Shearlet are considered as an extremely appropriate sparse directional image representation frame within MST (Multi-scale transform) theory so far [50]. These are affine systems with composite dilation of dimension $n=2$ and is explained as follows.

$$
\Psi_{j, l, k}(x)=|\operatorname{det} A|^{j / 2} \Psi\left(S^{l} A^{j} x-k\right)
$$

where $\left.\Psi \in L^{2} R\right)^{2}, A$ is an anisotropic matrix, that is related with scale transformation and $S$ is the shear matrix that is affiliated via area preserving geometrical transformation for example rotations and shear.

The scale, direction and shift parameter are denoted $j, l \& k$, respectively. For each $a>0 \& s \in R$, the matrices $A$ and $S$ plays the crucial role in the ST operation and represented as follows [51]

$$
A=\left[\begin{array}{cc}
a & 0 \\
0 & \sqrt{a}
\end{array}\right], \quad S=\left[\begin{array}{ll}
1 & s \\
0 & 1
\end{array}\right]
$$

If we assume that $a=4$ and $s=1$; we get

$$
A=\left[\begin{array}{ll}
4 & 0 \\
0 & 2
\end{array}\right], \quad S=\left[\begin{array}{ll}
1 & 1 \\
0 & 1
\end{array}\right]
$$


Let $A_{1}=\left[\begin{array}{ll}4 & 0 \\ 0 & 2\end{array}\right]$ and $S_{1}=\left[\begin{array}{ll}1 & 0 \\ 1 & 1\end{array}\right]$, for any $\xi=\left(\xi_{1}, \xi_{2}\right) \in R^{2}$ and $\xi_{1} \neq 0$; also let $\Psi^{(0)}$ and $\Psi^{(1)}$ is specified by

$$
\begin{aligned}
& \widehat{\Psi}^{(0)}(\xi)=\widehat{\Psi}^{(0)}\left(\xi_{1}, \xi_{2}\right)=\widehat{\Psi}_{1}\left(\xi_{1}\right) \widehat{\Psi}_{2}\left(\frac{\xi_{2}}{\xi_{1}}\right) \\
& \widehat{\Psi}^{(1)}(\xi)=\widehat{\Psi}^{(1)}\left(\xi_{1}, \xi_{2}\right)=\widehat{\Psi}_{1}\left(\xi_{1}\right) \widehat{\Psi}_{2}\left(\frac{\xi_{1}}{\xi_{2}}\right),
\end{aligned}
$$

Where $\widehat{\Psi}_{1}, \widehat{\Psi}_{2} \in C^{\infty}(\widehat{R})$, supp $\widehat{\Psi}_{1} \subset\left[-\frac{1}{2},-1 / 16\right] \cup[1 / 16,1 / 2]$, and supp $\widehat{\Psi}_{1} \subset[-1,1]$.

Each element of $\widehat{\Psi}_{j, l, k}$ is supported by the pair of trapezoids, approximately $2^{j} \times 2^{2 j}$ in size, orientated along slope lines $l 2^{-j}$

The ST function is then obtained:

$$
\begin{aligned}
& \Psi_{j, l, k}^{(0)}(x)=2^{\frac{3 j}{2}} \Psi^{(0)}\left(S_{0}^{l} A_{0}^{j} x-k\right) \\
& \Psi_{j, l, k}^{(1)}(x)=2^{\frac{3 j}{2}} \Psi^{(1)}\left(S_{1}^{l} A_{1}^{j} x-k\right),
\end{aligned}
$$

where $j \geq 0,-2^{j} \leq l \leq 2^{j}-1$, and $k \in Z^{2}$.

ST has the following characteristics: good spatial \& frequency localisation, robust anisotropic directionality selectivity, good parabolic scaling \& sparse representation. Still ST causes a Gibbs phenomenon due to the shortage of shift invariance. The version of shift invariant form of ST is NSST. A NSLP (nonsubsampled Laplacian pyramid) filters are utilised as a replacement for LP filters utilised in the ST operation. NSLP implements multi-scale decomposition. Each NSLP decomposition level can generate one low frequency \& one high frequency coefficient. Then, repeatedly, the next NSLP decomposition is conducted on the last (prior) low frequency coefficient in order to capture the singularities of an input image. When the decomposition level is aet at $J$, input image is decomposed into $J+1$ coefficients of equal size of the input image, one of that is the low frequency coefficient. To achieve multidirectional factorization, a shear filter (SF) is applied to the high frequency coefficients of each NSLP decomposition level without sub-sampling ensuring the shift invariance quality of NSST. Suppose, perform $l$ stages of directional decomposition on the high-frequency coefficient decomposes by NSLP, resulting in $2 l$ directional sub-bands of similar size as input [51]. Figure 2 depicts the two-level decomposition of the NSST. NSLP decomposition and their corresponding directional decomposition by SF are depicted in the schematic diagram in Figure 2.

\section{PROPOSED DENOISING METHOD}

This section presents a proposed denoising technique that combines NSST decomposition, NL-Means algorithm, and inverse NSST. The proposed denoising approach consists of three stages: NSST decomposition, NL-Means filtering of finer layers and NSST reconstruction. Figure 1 depicts the illustrative framework of proposed method. First, a NSST decomposition yields low and high frequency coefficients that reflect various feature information. The coarser elements of the input image contain low-frequency coefficient while the finer detailed features contain the high-frequency coefficients. The more visual significant details and contrast information can be found in the coarser layer of the images. More contour and edge information are provided by the finer layers of images. The finer layers are then processed using a nonlocal means algorithm, while the coarser layer is left as it is. It is possible to retain feature i.e. edges and structures of finer detailed layers using the nonlocal means algorithm. Finally, using inverse NSST to the base layer and the NLM filtered detail layers, a denoised image is obtained 


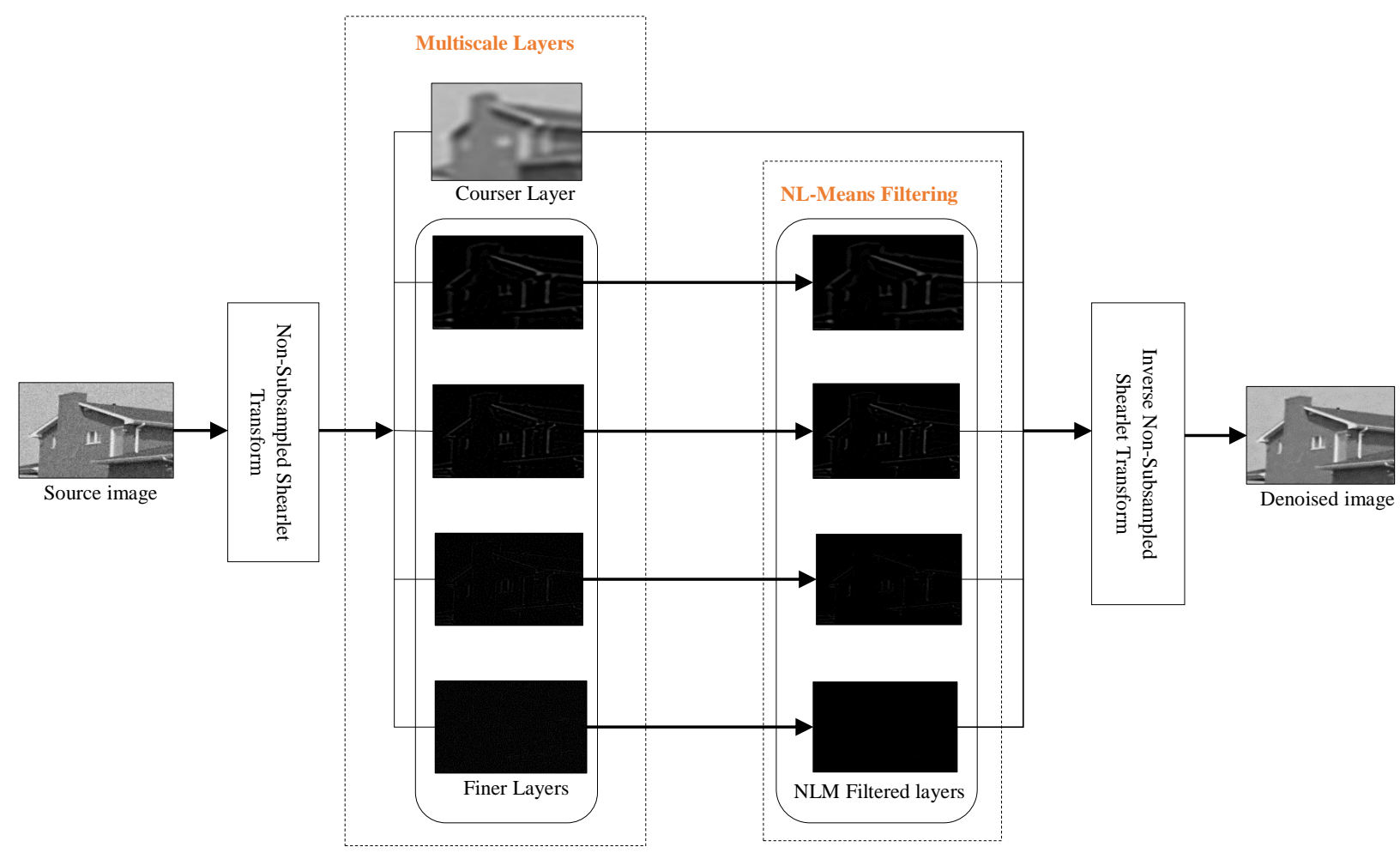

Fig.1. Framework of the proposed method

\subsection{NSST implementation steps}

The NSST could be achieved in two steps

(A) Multiscale decomposition: To achieve multi-resolution decomposition, a NSP (non-subsampled pyramid) filter bank decomposes each input image in the set of low \& high frequency sub images. Firstly, NSP decomposes input image into low frequency and high frequency coefficient. The singular points will be generated by iterating the NSP decomposition of each layer on the low frequency coefficients retrieved by upper layer decomposition. The sub band image will possess same size as input image if down-sampling is not performed. Finally, we get a low pass image and the $j$ band pass images from $j$ level decomposition.

(B) Directional localization; To achieve multi-direction decomposition, the sheralet filter bank decomposed this high frequency sub images. The pseudo polarisation coordinates are first converted to cartesian coordinates. A "Meyer" wavelet is then utilised to create a window function \& create shearlet filters. Lastly, the directional sub band images are obtained by convolving the sub band images with "Meyer" window function. Figure 2 indicates the two-level decomposition representation. The source image $A$ are decomposed by NSST in low frequency bands; $L_{A}(m, n)$ as well as high-frequency coefficient $H_{k, l}^{A}(m, n)$. The decomposed high frequency coefficient in the $k^{\text {th }}$ direction the $l^{\text {th }}$ decomposition level is denoted by $k$ and $l$. The domain variables of the NSST are indicated by letters $m$ and $n$. One low frequency coefficient and four high frequency coefficients of input image are achieved by the two-level NSST decomposition. 


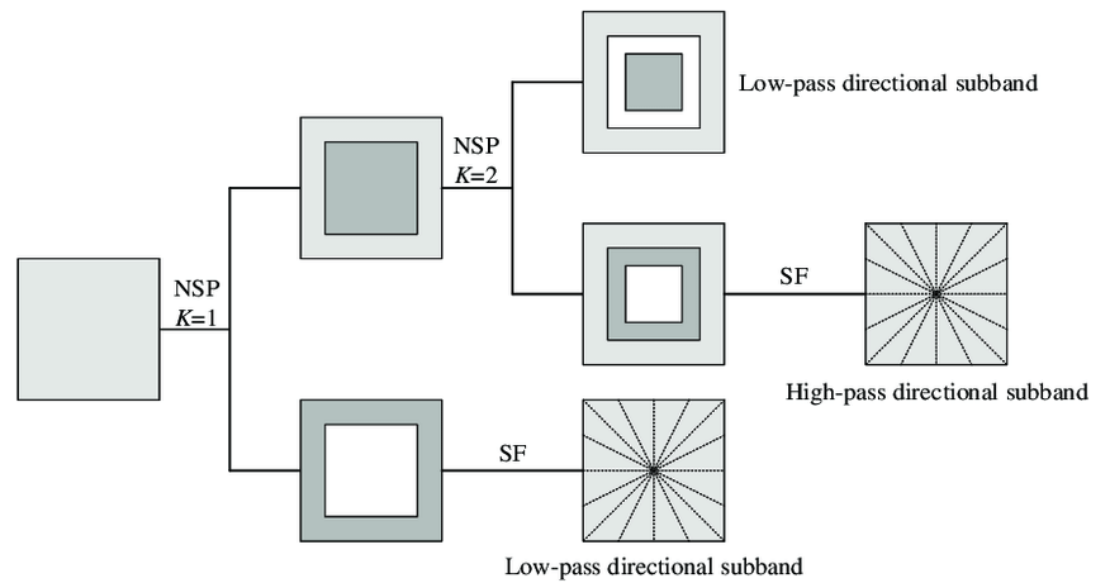

Fig. 2. Two level multiscale and multidirectional decomposition of NSST

\subsection{The Nonlocal means algorithm}

A nonlocal means algorithm [52] is the nonlinear, edge preserving filter which calculates each output pixels as the weighted sum of the input pixels. The set of input pixels which contribute to one output pixel will come from the large region of source image, hence it's called a nonlocal. The weights are computed by distance between small image patches which is the key property of nonlocal means filter. A nonlocal means filter is the variant of bilateral filter [53], that computes filter weights based on distance between pair of pixel values rather than small patches. Denoising performance is greatly enhanced as a result of this extension, and nonlocal means and its versions are among the mostly used denoising techniques.

The nonlocal means filter calculates the filtered vale $\hat{u}(p)$ of a pixel $p$ in a colour image $u=\left(u_{1}, u_{2}, u_{3}\right)$ as the weighted average of pixel in the square neighbourhood of size $2 r+1 \times 2 r+1$ centred on $p$, as defined by

$$
\hat{u}_{i}(p)=\frac{1}{C_{p}} \sum_{q \in N(p)} u_{i}(q) w(p, q)
$$

where $N(p)$ is the square neighbourhood centred on $p, w(p, q)$ represent weight contribution of $q$ to $p, i$ represent colour channel index and $C(p)$ represent normalisation factor,

$$
C(p)=\sum_{q \in N(p)} w(p, q) .
$$

A distance between the pair of small patches of size $2 f+1 \times 2 f+1$ centred at $p \& q$ is used to calculate the weight $w(p, q)$ of a neighbour $q$. The average of per-pixel and per-color channel squared distance $d_{i}^{2}(p, q)$ over the patches is called the patch distance $d^{2}(P(p), P(q))$.

$$
\begin{gathered}
d_{i}^{2}(p, q)=\left(u_{i}(p)-u_{i}(q)\right)^{2} \\
d^{2}(P(p), P(q))=\frac{1}{3(2 f+1)^{2}} \sum_{i=1}^{3} \sum_{n \in P(0)} d_{i}^{2}(p+n, q+n)
\end{gathered}
$$

Here $P(0)$ indicates the offset to each pixel within a patch, $P(p) \& P(q)$ are the patches centred on $p$ and $q$, respectively. The computed squared distance is biased due to the noisy input image, which is an important observation. As a result, the original nonlocal means filter subtracts the variance of the computed squared distance from the patch distance to remove the noise contributions. A modified patch distance is calculated using uniform pixel noise with variance $\sigma^{2} \&$ uncorrelated pixels $p \& q$ as

$$
\max \left(0, d^{2}(P(p), P(q))-2 \sigma^{2}\right)
$$

An exponential kernel is then used to calculate the weight $w(p, q)$ of the contribution of pixel $q$ to $p$ as 


$$
w(p, q)=\exp ^{-\frac{\max \left(0, d^{2}(P(p), P(q))-2 \sigma^{2}\right)}{k^{2} 2 \sigma^{2}}}
$$

Where $k$ is a damping factor set by the user to control the strength of filter. A more conservative filter is produced with a lower $k$ value.

We also employ the patchwise extension described by [54], which results in outputs that are slightly smoother. We weight complete pixels in the patch centred at $p$ with $w(p, q)$ in place of weighting only the pixel $p$ at the centre of patch with the weight $w(p, q)$. Each pair of pixels appears as $2 f+1 \times 2 f+1$ patches. Every time with the different weight $w(p+n, q+n)$, where $n$ is the offset of $p$ and $q$ in the patch. A final weight $W(p, q)$ for the pair of pixels in the patchwise implementation is just the average of complete weights which involve these two pixels;

$$
W(p, q)=\frac{1}{(2 f+1)^{2}} \sum_{n \in P(0)} w(p+n, q+n)
$$

The value of $\sigma$ determines the size of the patch and research window. When $\sigma$ increases, we need a larger patch to ensure that patch comparison is reliable. At the same time, we need to enhance the research window to boost the algorithm's noise-removal capabilities by locating more similar pixels. Where $h=k \sigma$ is the value of the filtering parameter. As the dimension of the patch increases, the value of $\sigma$ drops. The distance between two pure noise patches concentrates more around $2 \sigma^{2}$ for larger sizes, and hence a smaller value of $k$ can be utilised for filtering.

\subsection{NSST Reconstruction}

In order to generate a final denoised image, inverse NSST is applied to base layer and the NLM filtered detail layers.

\subsection{Performance metrices}

The method of evaluation is required to demonstrate the effectiveness of the denoising methods. Subjective and objective assessment methods are two types of evaluation procedures that are often used. Qualitative approaches are manmade visual analysis that aids in describing the visual quality of the images. There are variety of strategies that can be employed for objective analysis [40]. The PSNR and its Mean value are quantitative evaluation metrics estimated for measuring the effectiveness of denoised image reconstructed in our method. The PSNR is expressed in decibels. The larger the PSNR value, the greater the quality of the resulting denoised image. It is computed as follows:

$$
P S N R=10 \log _{10}\left(\frac{M A X_{I}^{2}}{M S E}\right)
$$

Where $M A X_{I}$ indicates the maximum possible pixel value of an image. The cumulative error between an original image and denoised image is called mean squared error (MSE). The lower the estimated MSE value, the superior the image denoising performance. It is computed as follows:

$$
M S E=\frac{1}{n} \sum_{i=1}^{n}\left(Y_{i}-\widehat{Y}_{i}\right)^{2}
$$

The vector $n$ predictions is derived from the sample on $n$ data points on all variables $\& Y$ is the vector of observed values of the variable being predicted, with $\hat{Y}$ being the predicted values.

To further assess the consistency of the different algorithms, we introduce calculating the mean/average of PSNR values, which may be computed as follows,

$$
\text { Mean }=\sum_{i=0}^{n} \frac{P S N R_{i}}{n}
$$


Above metric can be used to determine the stability in the performance of the of different algorithms at various noise levels.

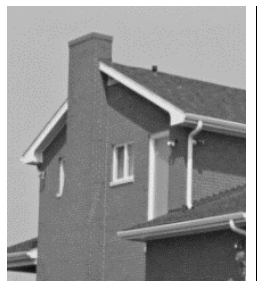

(a)

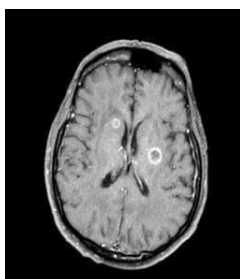

(b)

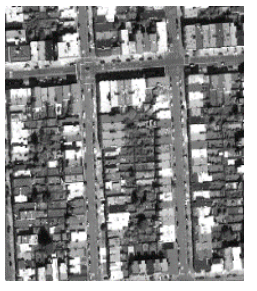

(c)

Fig. 3. Input images (a) House; (b) MRI; (c) PAN

\section{EXPERIMENTAL SETUP, RESULTS AND DISCUSSION}

\section{(A) Experimental setup}

With the aim to test the effectiveness of our denoising method, we have utilized three images: house image, magnetic resonance imaging (MRI) image and panchromatic (PAN) image that is remote sensing, medical and natural image, respectively [55]. We had evaluated the effectiveness of our denoising method with different set of images. The images are of size $256 * 256$ pixels. The efficiency of our method was evaluated utilizing standard 8-bit gray scale image contaminated by zero mean white gaussian noise. The proposed method has been performed in MATLAB 2019b on intel (R) core (TM) i3-7020 CPU @ $2.70 \mathrm{GHz}$ system with 8 GB memory. An effectiveness of our method is compared with NLGRTV [30], Locally Adaptive Kernel Regression (LARK) [31], Total variation minimization [32], Bitonic filter [33], GBFMT [34], NLFMT [35], RBF [36], Markov Random Field (MRF) [37] and SBF [38]. For this experiment, a radius of the aussian filter is set to 3 (three), however as larger value could induce excessive smoothing \& blurring. For SBF, the spatial kernel parameter is set to 4 (four)

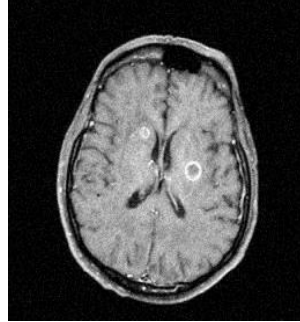

$\sigma=10$

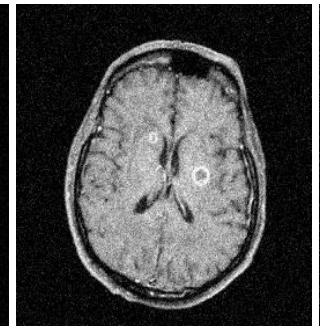

$\sigma=20$

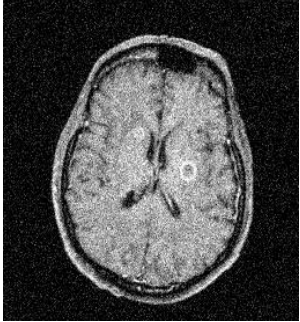

$\sigma=30$

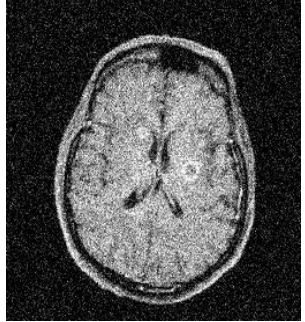

$\sigma=40$

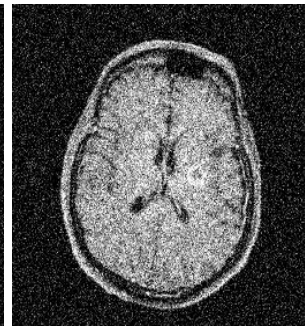

$\sigma=50$

Fig.4. A source MRI image contaminated by gaussian noise on $\sigma$ (standard deviation) = 10,20,30,40,50

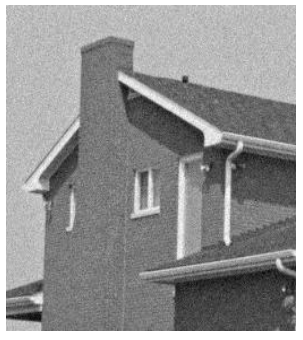

$\sigma=10$

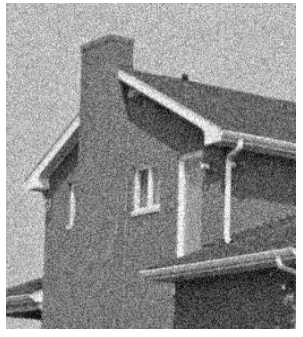

$\sigma=20$

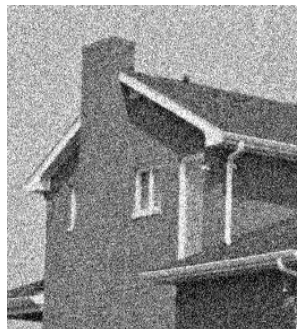

$\sigma=30$

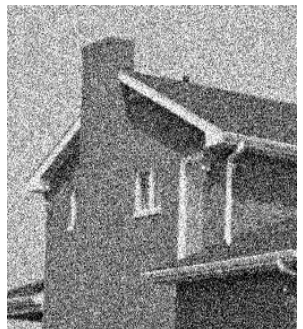

$\sigma=40$

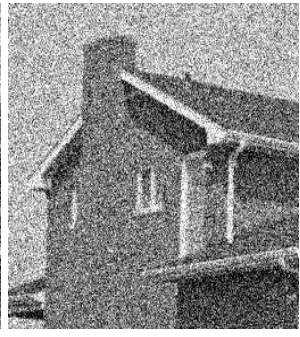

$\sigma=50$

Fig.5. A source House image contaminated by gaussian noise on $\sigma$ (standard deviation) $=10,20,30,40,50$
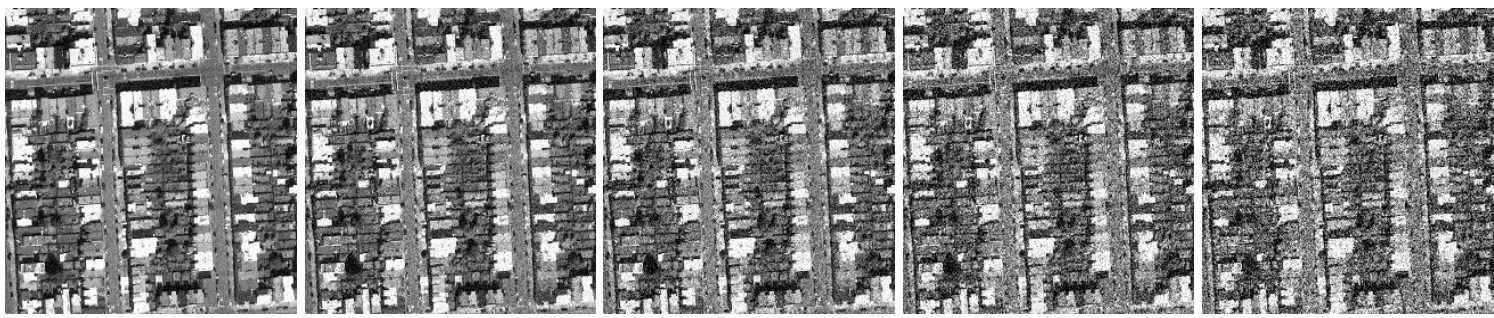
Fig.6. A source PAN image contaminated by gaussian noise on $\sigma$ (standard deviation) =10,20,30,40,50

and a kernel range parameter is set at 20. The performance of our method is measured for $\sigma=10,20,30,40,50$ for low to high noise levels. The standard test images are shown in Figure 3. These images are then added with white gaussian noise of difference variance. The noisy image for MRI, House, and PAN are shown in Figures 4, 5 , and 6 at various levels of noise.

\section{(B) Experimental results}

This section demonstrates an experimental results achieved by processing the standard test image datasets used in our algorithm and results are compared visually as well as quantitatively with several algorithms such as NLGRTV (nonlocal version of general relative total variation), Locally Adaptive Kernel Regression (LARK), Total variation minimization, Bitonic filter, NLFMT, GBFMT, RBF, Markov Random Fields (MRF), SBF and proposed method and proposed method. The results are provided for all three datasets $\&$ five distinct noise give the comparative visual analysis and quantitative results at various settings. The qualitative evaluation results for the MRI at $\sigma=$ 10 is shown in Fig 7 (a-j). Figures 8 to 11 demonstrate the findings of MRI using various algorithms with noise levels of $\sigma=20,30,40$ and 50. The visual findings for the house image at $\sigma=10,20,30,40$ and 50 utilizing various approaches are shown in Figure 12 to 16. Similarly, Figure 17 to 21 demonstrates the denoised performance of PAN image at $\sigma=10,20,30,40$ and 50, respectively using various algorithms. Table 1 demonstrates the objective performance with regard to PSNR values at $\sigma=10,20,30,40$ and 50 utilizing the NLGRTV, LARK, Total variation minimization, Bitonic filter, NLFMT, GBFMT, RBF, Markov Random Fields (MRF), SBF and proposed method and our proposed algorithm for MRI image, PAN image \& House image

\section{(C) Result analysis and discussions}

We have demonstrated the qualitative and quantitative evaluations of our proposed algorithm in this section and compare it with other algorithms.

\section{(i) Qualitative Evaluation}

We have evaluated the proposed denoising algorithm with PSNR value, mean value and visual human perception after implementing it. In order to evaluate our findings, we considered three images: MRI, House, and PAN. Despite the fact that PSNR measures the intensity differences between the images, qualitative analysis plays the prominent role in order to verify and validate the visual quality of the denoised image. of image quality is crucial for qualitative judgement. It aids in identifying whether or not edge features are retained, to examine either images carry artifacts and either noise has been eliminated. From Figures 7 to 21, it is noticeable that our method surpasses other prevailing methods in the context of qualitative analysis at standard deviations of $\sigma=10,20,30$. Therefore at low to moderate levels such as at standard deviation $\sigma=10,20$ and 30, our method retained edges and sharp gradients, also tips are crisper, boundaries and counters are well sharp after removing noise. Therefore, practically at low to moderate noise levels, our method performs better then prevailing methods. As a result, proposed method has performed better visual performance then other benchmarking methodologies such as NLGRTV, LARK, Total variation minimization, Bitonic filter, NLFMT, GBFMT, RBF, Markov Random Fields (MRF), SBF. However, NLFMT, GBFMT, MRF, and SBF have performed quite similar visual performance at low to moderate noise levels as compares to proposed method but rest of the approaches failed miserably in maintaining fine textures and causes over-blurring of the images, resulting in data loss. A proposed method produces overall better visual results when compared to other algorithms and simultaneously emerges as a preferable algorithm for quality performance at low to moderate noise levels. Our method performs nearly better performance as compares to other benchmarking methods. Despite the fact that the algorithm was created to deal with noise at moderate and high noise levels, but our method is efficient to obtain comparable results at low noise levels too. The following factors are responsible for the improving the performance of proposed method i.e. nonsubsampled shearlet transform (NSST) has potential to captures the geometry of multidimensional information. Shear parameters are than used to capture the singularities. The non-local means filter reduces noise in detail images while maintaining the sharpness of strong edges, such as the image

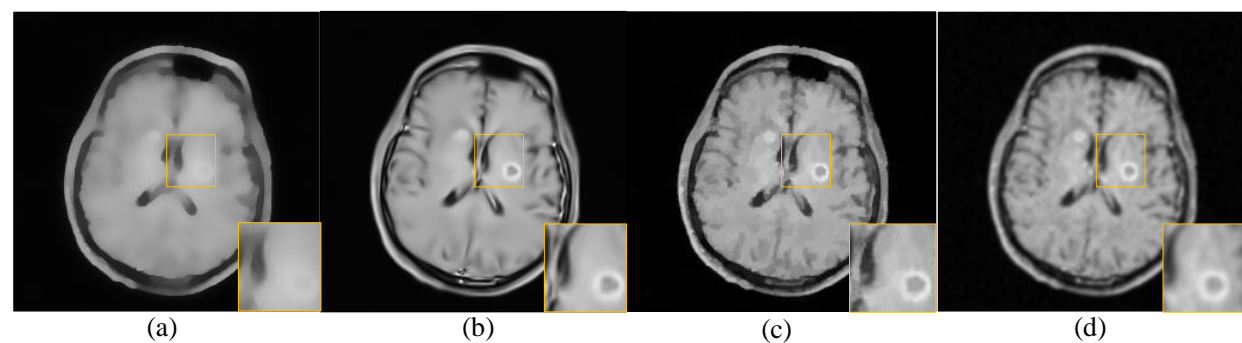

(a)

(b)

(c)

(d)

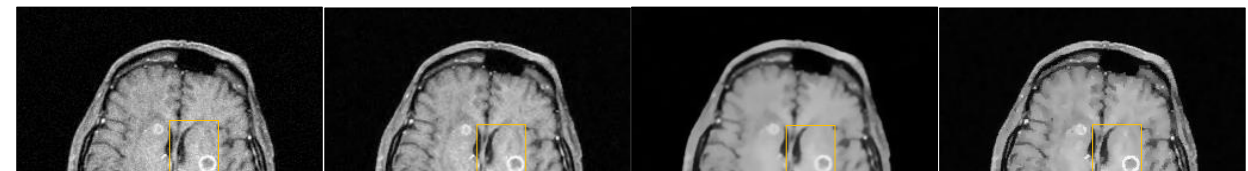


Fig. 7. MRI image $\sigma=10$, denoising result (a) NLGRTV, (b) Ker. Reg., (c) TV1, (d) Bitonic, (e) NLFMT, (f) GBFMT, (g) RBF, (h) MRF, (i) SBF, (j) Our method
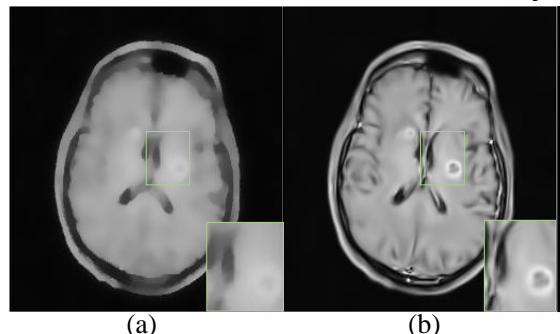

(b)

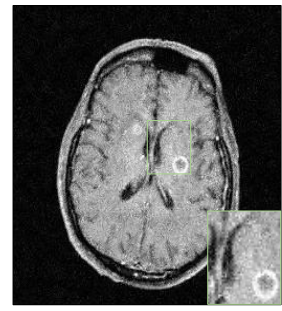

(e)

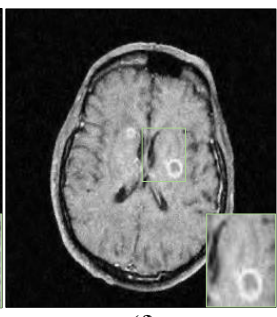

(f)

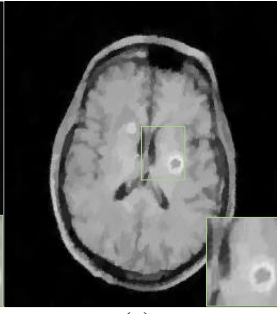

(c)

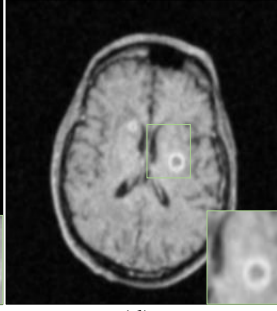

(d)

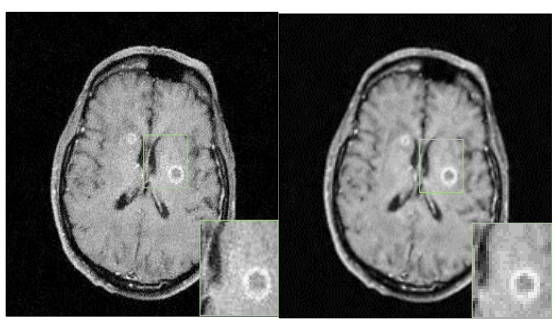

(i)

(j)

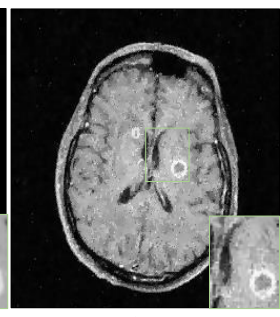

(h)

Fig. 8. MRI image at $\sigma=20$, denoising result (a) NLGRTV, (b) Ker. Reg., (c) TV1, (d) Bitonic, (e) NLFMT, (f) GBFMT, (g) RBF, (h) MRF, (i) SBF, (j) Our method 


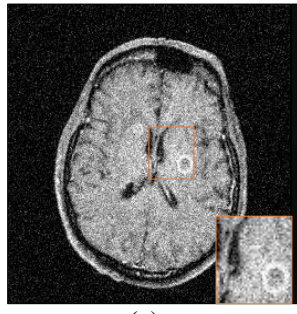

(a)

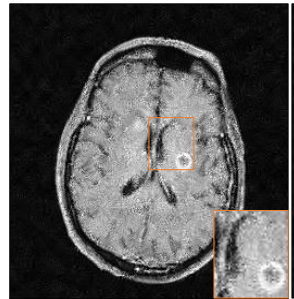

(e)

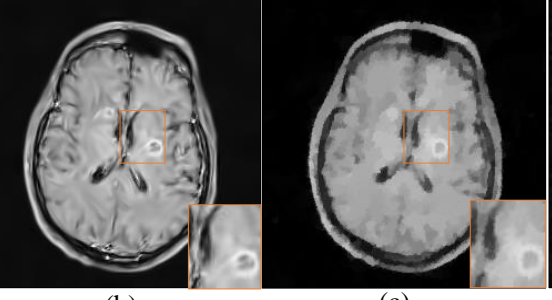

(c)

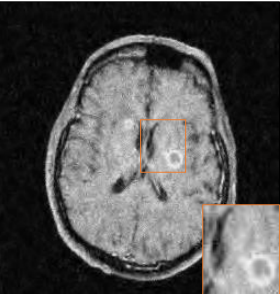

(f)

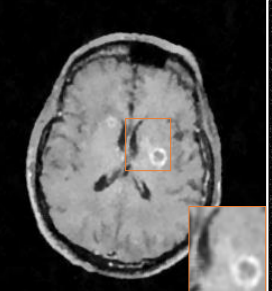

(g)

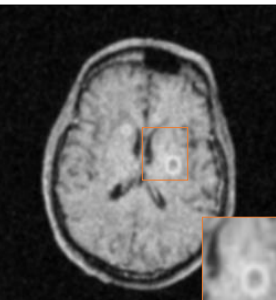

(d)

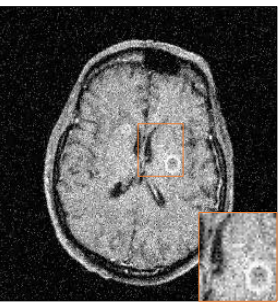

(h)

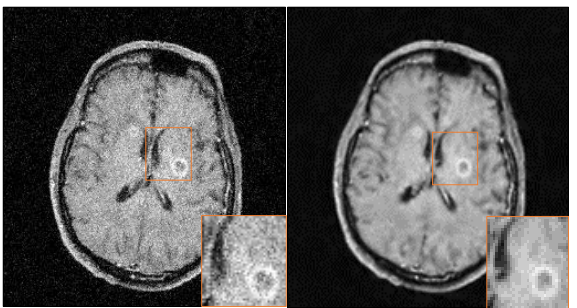

(i)

(j)

Fig. 9. MRI image at $\sigma=30$, denoising result (a) NLGRTV, (b) Ker. Reg., (c) TV1, (d) Bitonic, (e) NLFMT, (f) GBFMT, (g) RBF, (h) MRF, (i) SBF, (j) Proposed

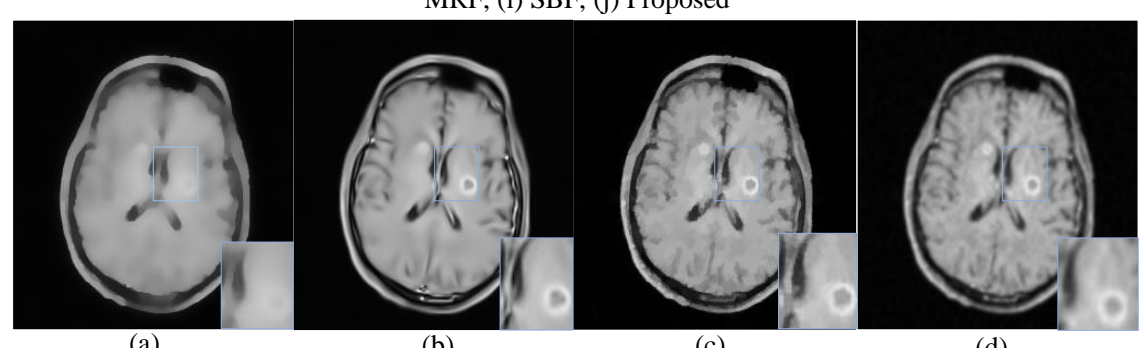

(a)

(b)

(c)

(d)

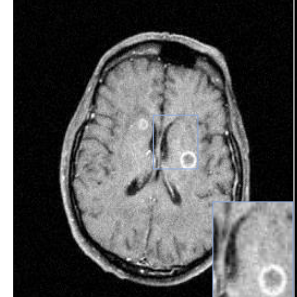

(e)

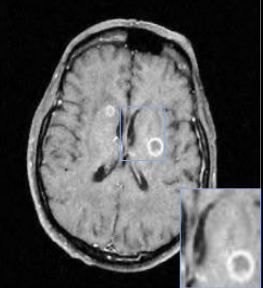

(f)

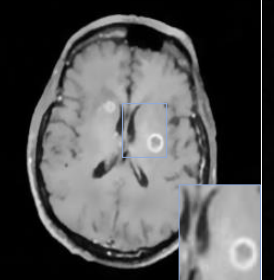

(g)

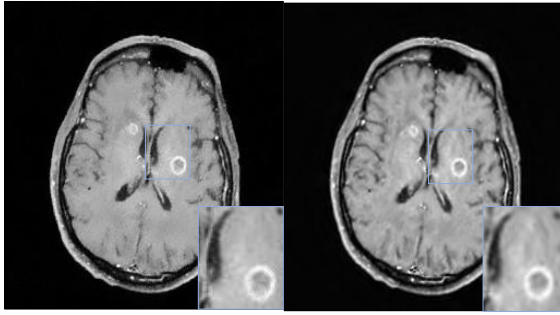

(j)

(i)

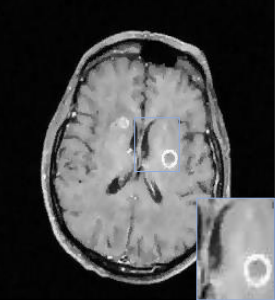

(h)

Fig.10. MRI image at $\sigma=40$, denoising result (a) NLGRTV, (b) Ker. Reg., (c) TV1, (d) Bitonic, (e) NLFMT, (f) GBFMT, (g) RBF, (h) MRF, (i) SBF, (j) Our method 


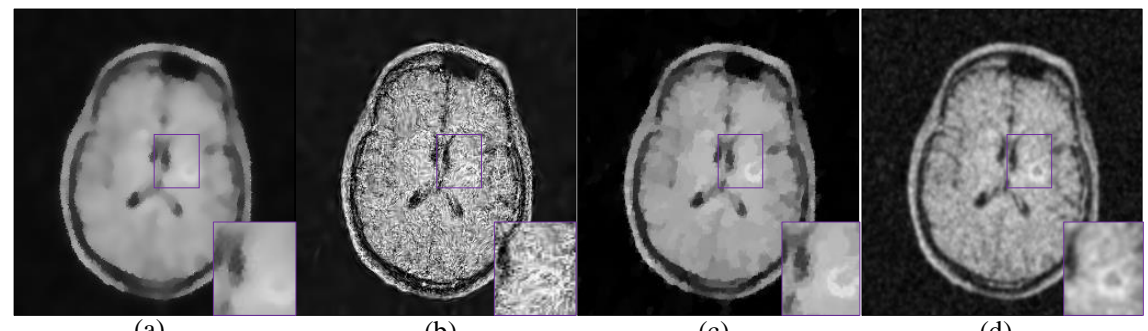

(a)

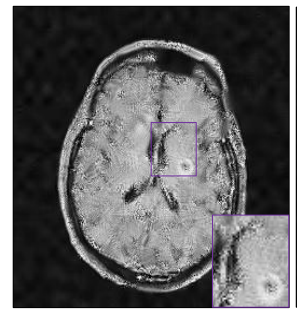

(e)

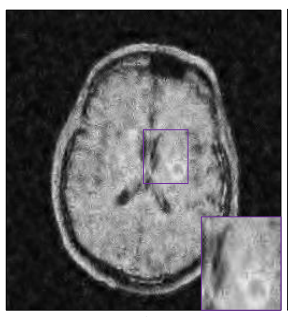

(f) (c)

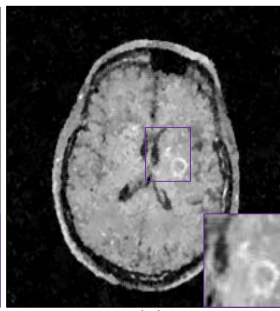

(g)

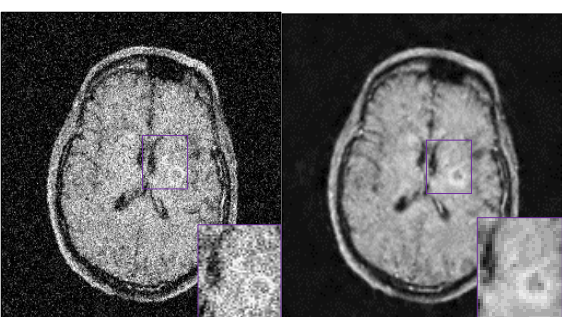

(j)

(i)

(d)

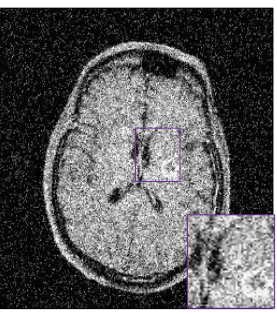

(h)

Fig.11. MRI image at $\sigma=50$, denoising result (a) NLGRTV, (b) Ker. Reg., (c) TV1, (d) Bitonic, (e) NLFMT, (f) GBFMT, (g) RBF, (h) MRF, (i) SBF, (j) Proposed

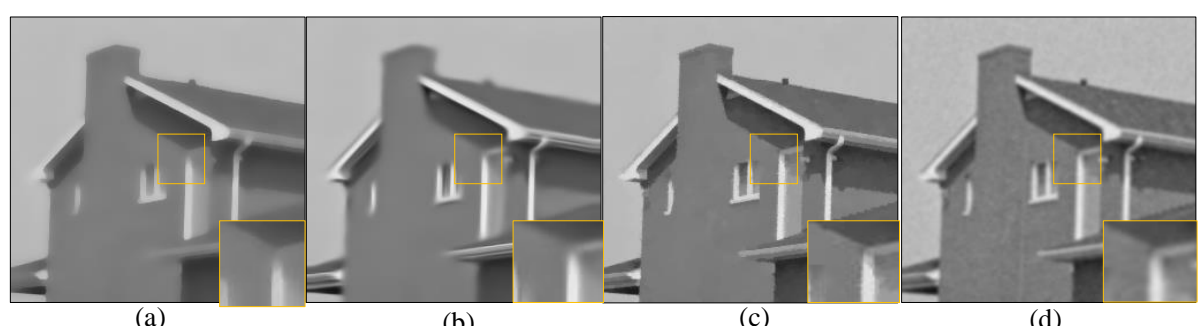

(a)

(b)

(d)

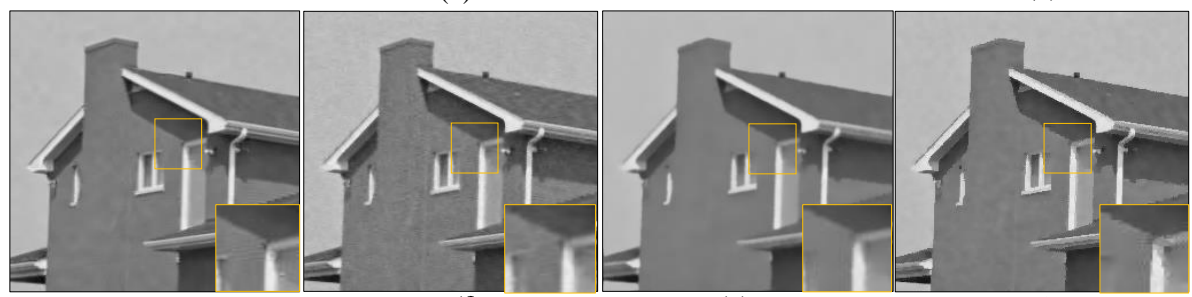

(e)

(g)

(h)

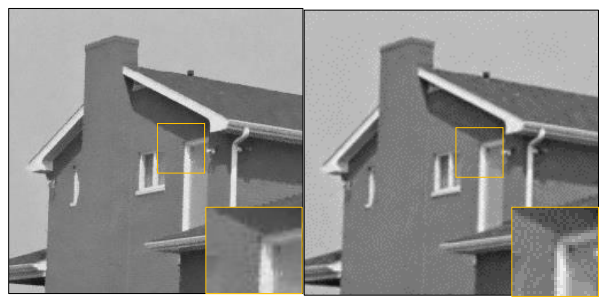

(i)

(j)

Fig.12. House image at $\sigma=10$, denoising result (a) NLGRTV, (b) Ker. Reg., (c) TV1, (d) Bitonic, (e) NLFMT, (f) GBFMT, (g) RBF, (h) MRF, (i) SBF, (j) Our method 


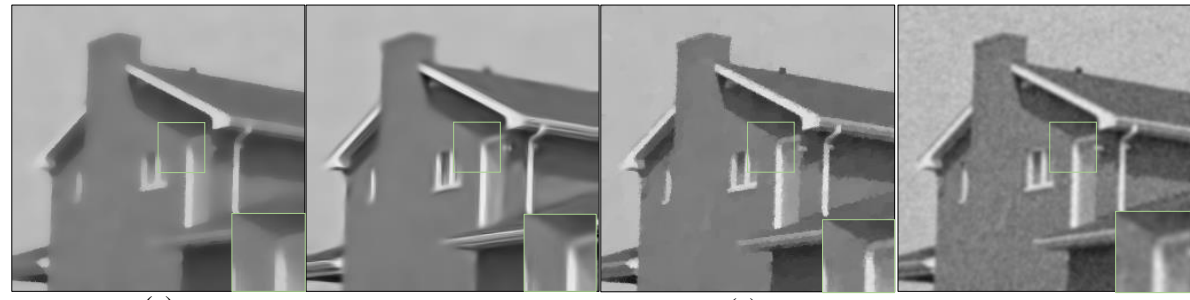

(a)

(b)

(c)

(d)

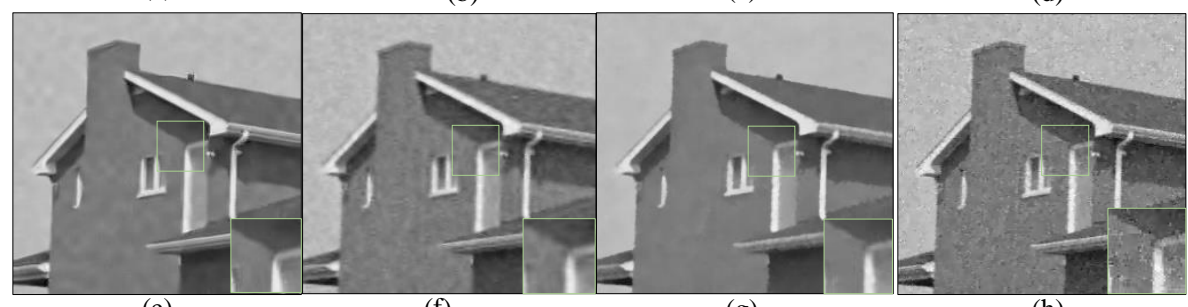

(e)

(f)

$(\mathrm{g})$

(h)

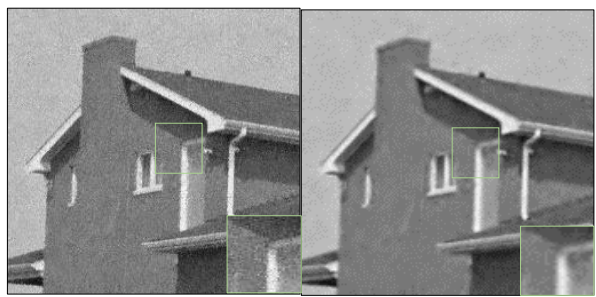

(i)

(j)

Fig.13. House image at $\sigma=20$, denoising result (a) NLGRTV, (b) Ker. Reg., (c) TV1 (d) Bitonic, (e) NLFMT, (f) GBFMT, (g) RBF, (h) MRF, (i) SBF, (j) Proposed

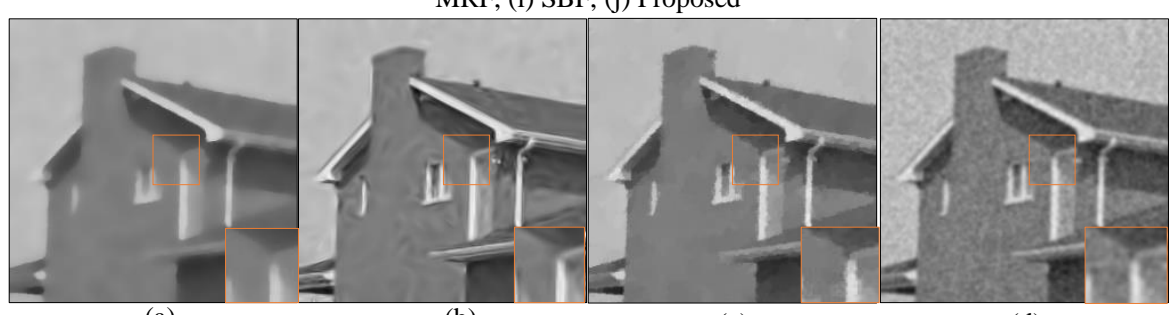

(a)

(b)

(c)

(d)

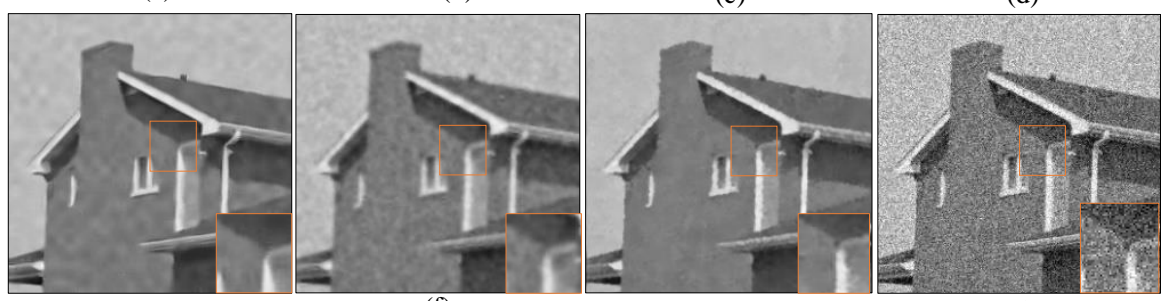

(e)

(g)

(h)

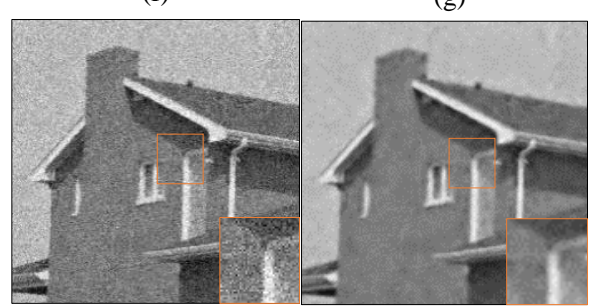

(i)

(j)

Fig.14. House image at $\sigma=30$, denoising result (a) NLGRTV, (b) Ker. Reg., (c) TV1, (d) Bitonic, (e) NLFMT, (f) GBFMT, (g) RBF, (h) MRF, (i) SBF, (j) Our method 


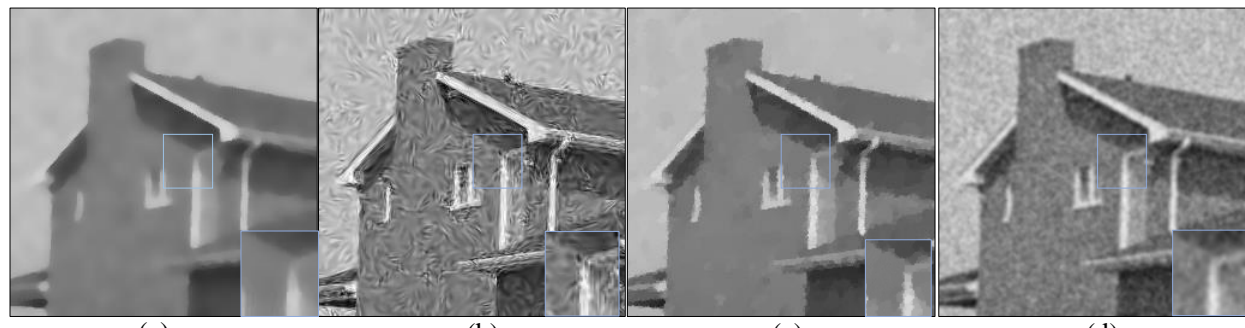

(a)

(b)

(c)

(d)

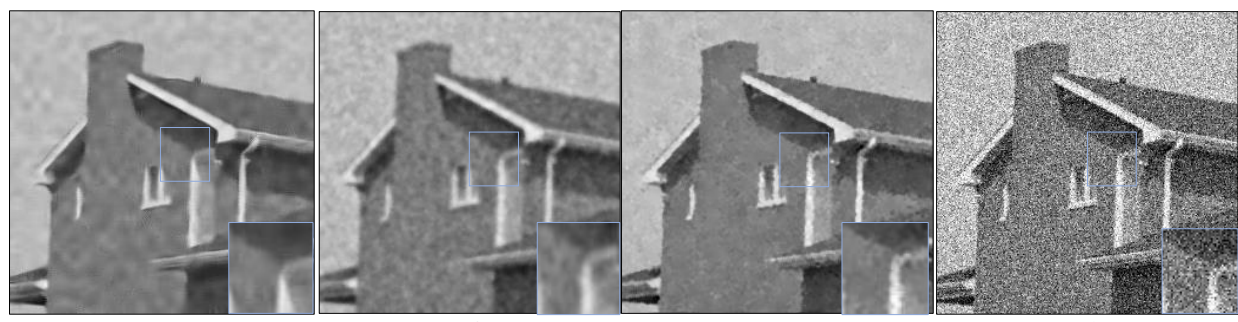

(e)

(g)

(h)

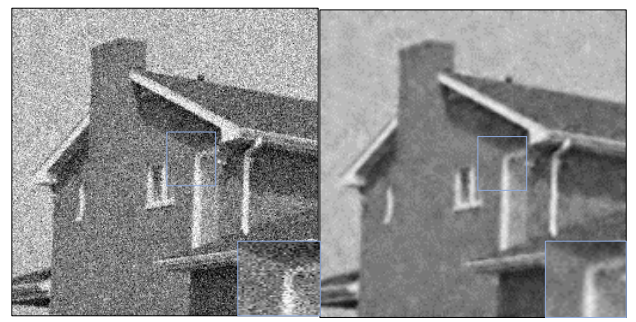

(i)

(j)

Fig.15. House image at $\sigma=40$, denoising result (a) NLGRTV, (b) Ker. Reg., (c) TV1, (d) Bitonic, (e) NLFMT, (f) GBFMT, (g) RBF, (h) MRF, (i) SBF, (j) Our method

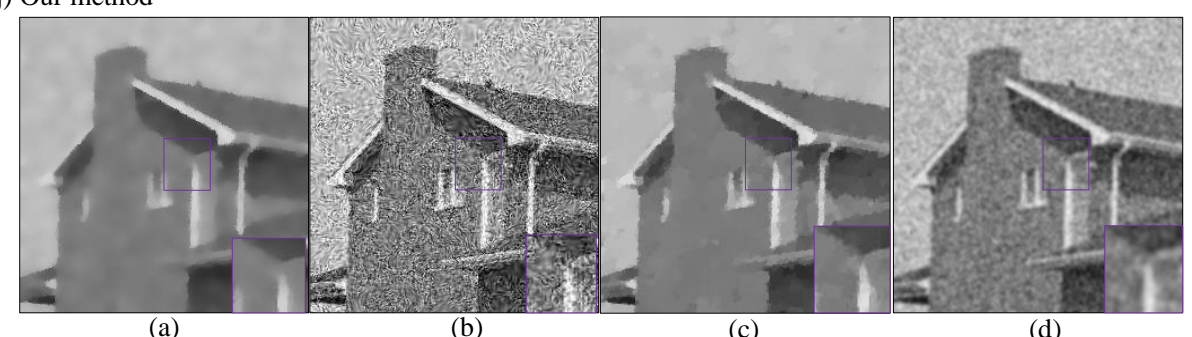

(a)

(b)

(c)

(d)

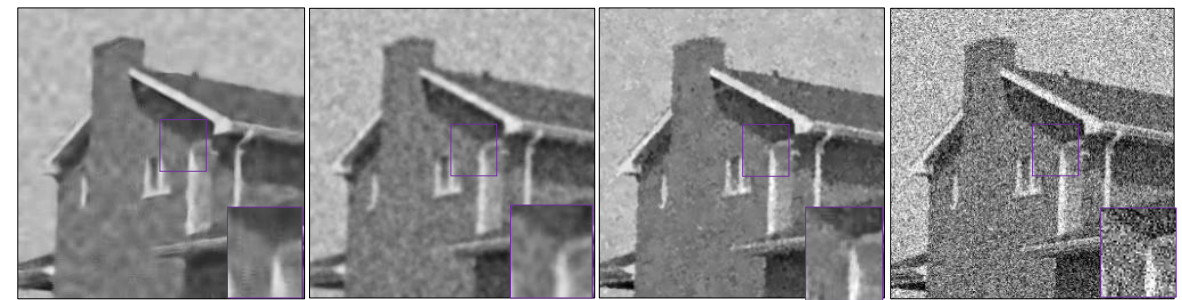

(e)

(g)

(h)

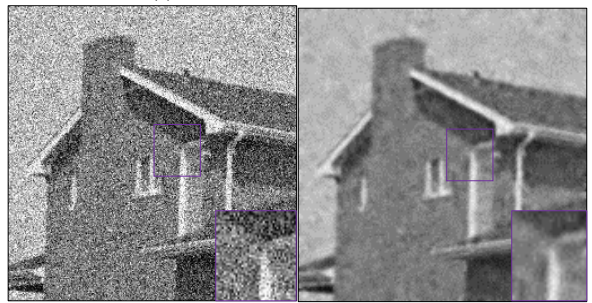

(i)

(j)

Fig.16. House image at $\sigma=50$, denoising result (a) NLGRTV, (b) Ker. Reg., (c) TV1, (d) Bitonic, (e) NLFMT, (f) GBFMT, (g) RBF, (h) MRF, (i) SBF, (j) Our method 

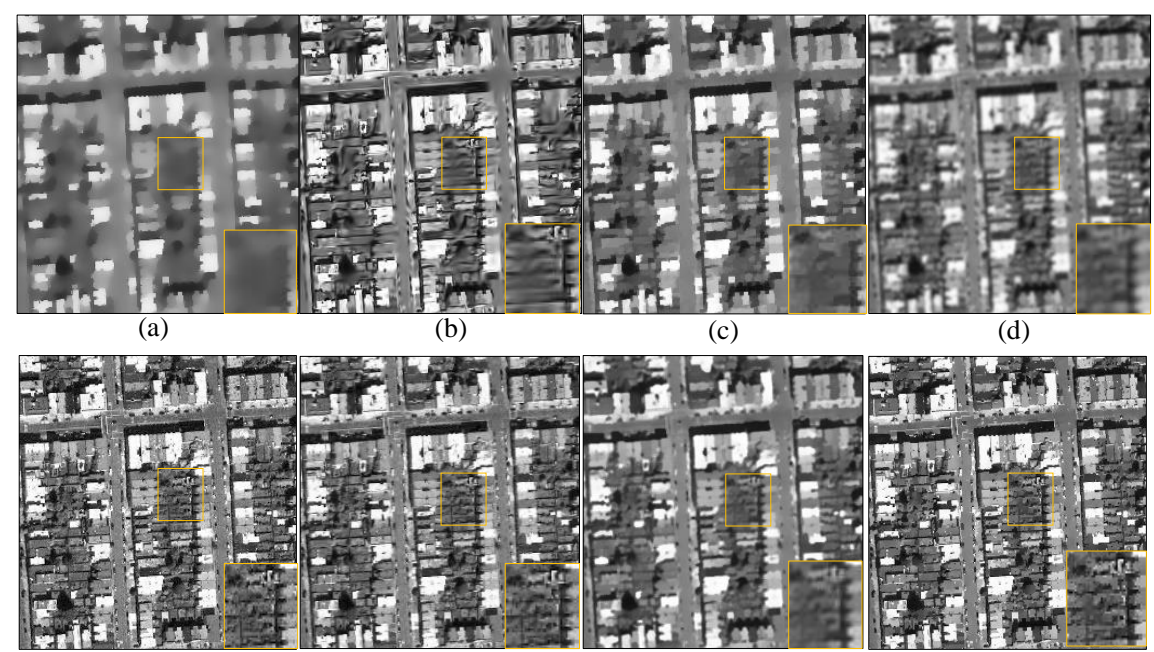

(e)

(f)

(g)

(h)

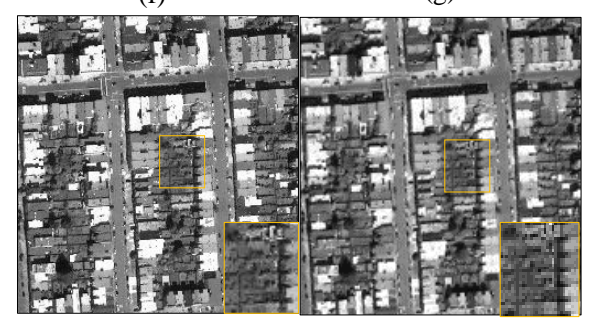

(i)

(j)

Fig.17. PAN image at $\sigma=10$, denoising result (a) NLGRTV, (b) Ker. Reg., (c) TV1, (d) Bitonic, (e) NLFMT, (f) GBFMT, (g) RBF, (h) MRF, (i) SBF, (j) Our method

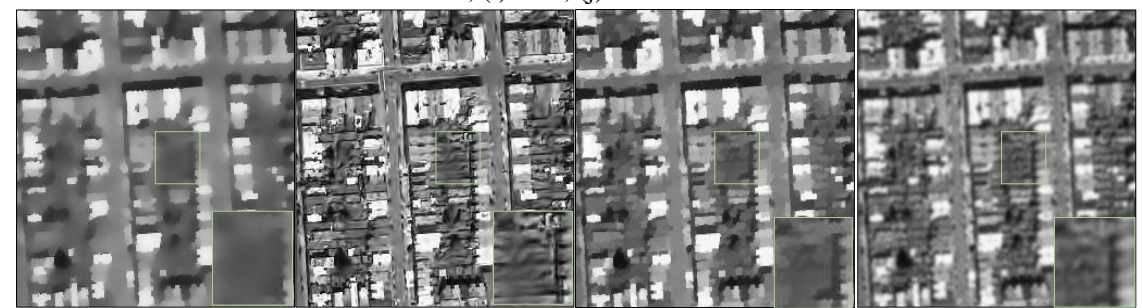

(a)

(b)

(c)

(d)

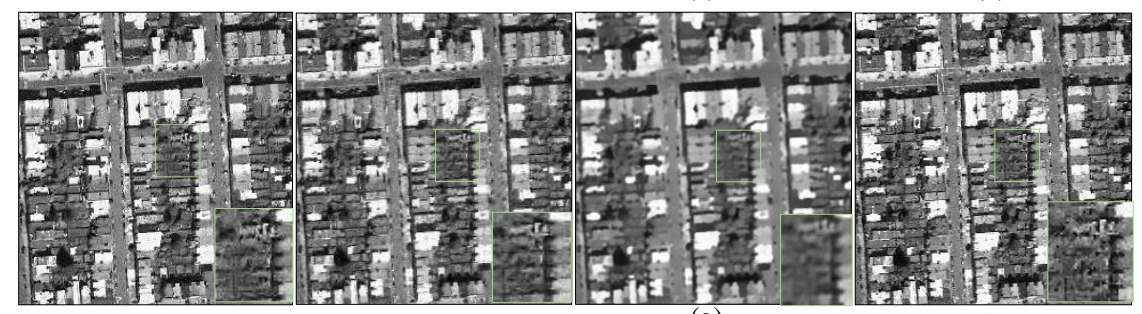

(e)

(g)

(h)

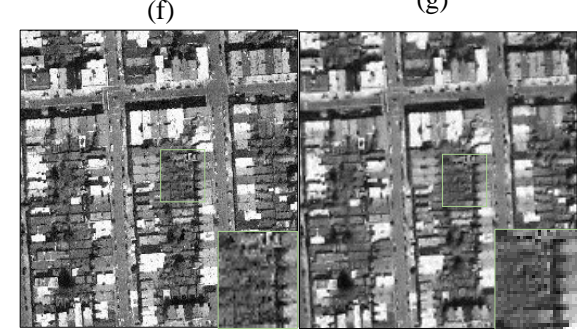

(i)

(j)

Fig.18. PAN image at $\sigma=20$, denoising result (a) NLGRTV, (b) Ker. Reg., (c) TV1, (d) Bitonic, (e) NLFMT, (f) GBFMT, (g) RBF, (h) MRF, (i) SBF, (j) Our method 


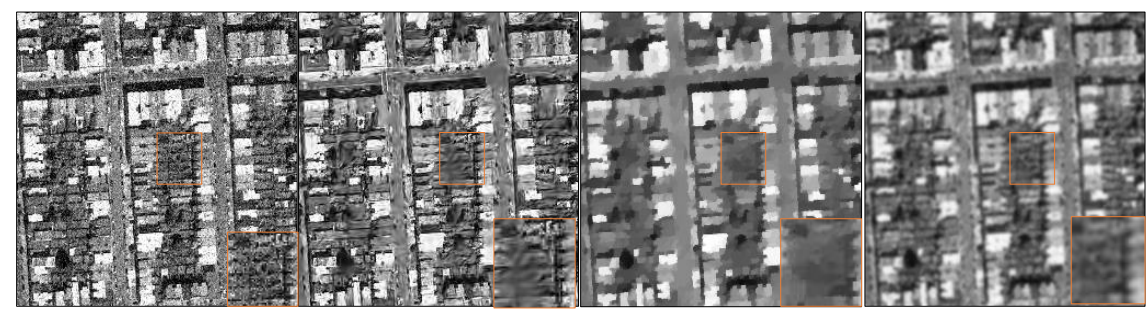

(a)

(b)

(c)

(d)

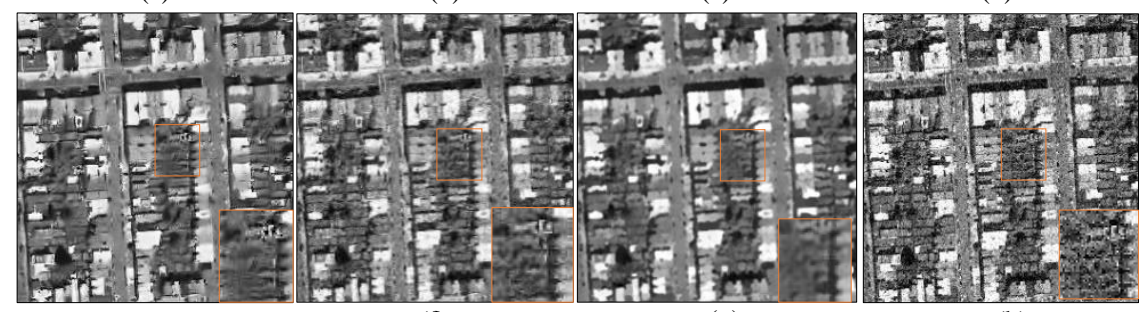

(e)

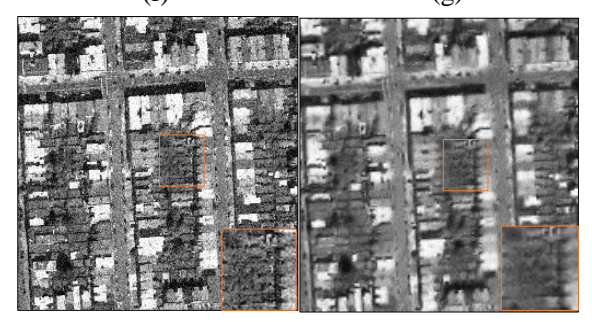

(h)

(i)

(j)

Fig.19. PAN image at $\sigma=30$, denoising result (a) NLGRTV, (b) Ker. Reg., (c) TV1, (d) Bitonic, (e) NLFMT, (f) GBFMT, (g) RBF, (h) MRF, (i) SBF, (j) Our method

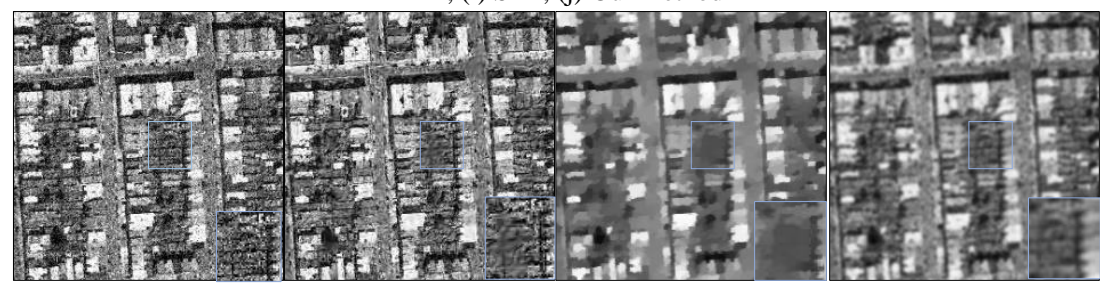

(a)

(b)

(c)

(d)

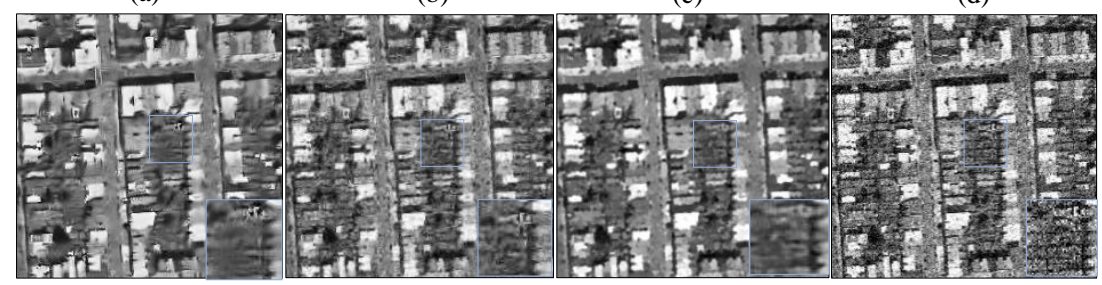

(e)

(g)

(h)

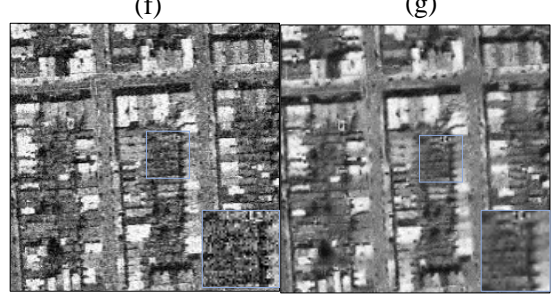

(i)

(j)

Fig.20. PAN image at $\sigma=40$, denoising result (a) NLGRTV, (b) Ker. Reg., (c) TV1, (d) Bitonic, (e) NLFMT, (f) GBFMT, (g) RBF, (h) MRF, (i) SBF, (j) Our method 


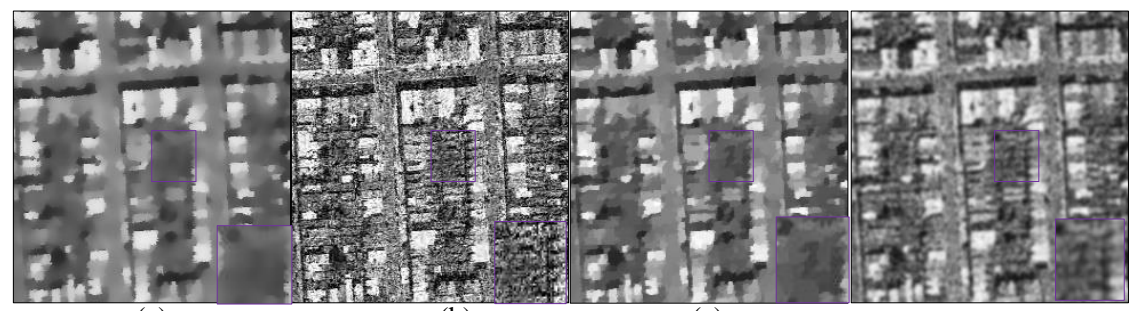

(a)

(b)

(c)

(d)

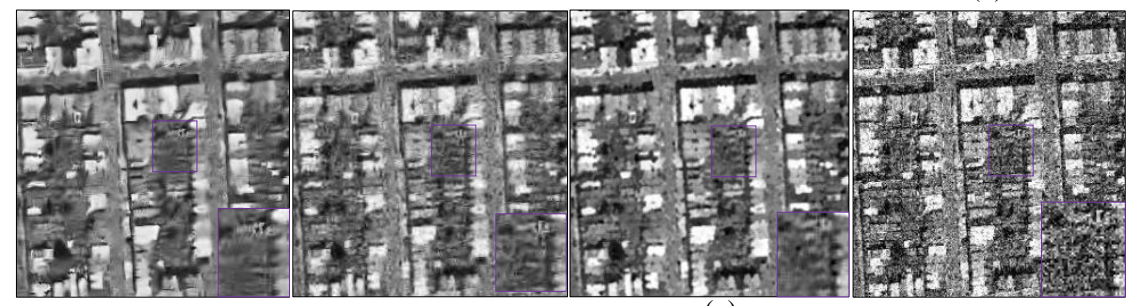

(e)

(f)

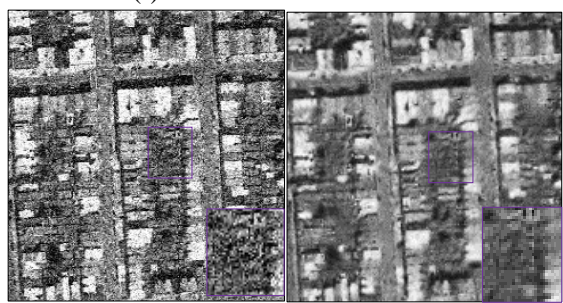

(i)

(j)

Fig.21. PAN image at $\sigma=50$, denoising result (a) NLGRTV, (b) Ker. Reg., (c) TV1, (d) Bitonic, (e) NLFMT, (f) GBFMT, (g) RBF, (h) MRF, (i) SBF, (j) Proposed

silhouette. When compared to noisy images, this filter also smooths textured regions, resulting in more information. The combined technique can able to combine the benefits of efficient algorithms having exceptional ability to extract multidimensional data geometry. It could also effectively present edges in low-noise for any given image. Bitonic filter and RBF perform roughly identical and somewhat better in terms of visual perception, but with insufficient noise removal and loss of fundamental feature information. Bitonic outperforms Ker, Reg, TV1, and NLGRTV algorithms because it removes high amount of noise from the images. The proposed approach, on the other hand, was able to totally eradicate the noisy pixels and maintains sharp edges and smooth gradients. We zoomed in on different regions of an image to check the fine texture details of denoised image and noticed that the texture details are more clearly visible in the images in proposed algorithm, however while zooming images in other methods result in presence of noise pixels instead of texture details. As a result, the proposed method may be concluded that, it maintains better performance than other comparative algorithms in case of qualitative assessment.

\section{(ii) Quantitative Evaluation}

In order to assess the effectiveness of our method quantitatively, we have computed PSNR value and its mean value for the test image datasets. The quantitative assessment of our method along with other methods is illustrated in Table 1. As shown in Table 1, the objective metric values of our algorithm are generally greater than other comparative algorithms. The PSNR values clearly shows that proposed algorithm performs better at moderate to high noise levels from the Table 1. Whereas the results of proposed algorithm are slightly better at low noise level, meanwhile still appropriate and effective as compares to other existing algorithms. Given that the noise levels of the image to be denoised is undefined beforehand, therefore selecting a good denoising filter only due to PSNR performance is not reasonable. In order to overcome this problem, we propose to measure the mean value of PSNR, which exhibits the stability in the performance of different algorithms across various range of noise levels. Table 1 shows that proposed algorithm is capable to provide superior results for House, MRI and PAN image based on the objective metrics. The proposed approach and SBF both perform well on PAN

Table 1: Quantitative assessment in terms of PSNR and Mean value for House, MRI and PAN image with noise levels of $\sigma=10,20,30,40$ and 50 


\begin{tabular}{|c|c|c|c|c|c|c|}
\hline Datasets & Methods & $\sigma=10$ & $\sigma=20$ & $\sigma=30$ & $\sigma=40$ & $\sigma=50$ \\
\hline \multirow{10}{*}{ MRI } & NLGRTV [30] & 24.12 & 23.71 & 20.14 & 22.54 & 22.27 \\
\hline & Ker. Reg. [31] & 28.45 & 27.63 & 26.01 & 22.86 & 18.91 \\
\hline & TV1[32] & 28.71 & 27.04 & 25.96 & 23.99 & 21.21 \\
\hline & Bitonic [33] & 29.39 & 27.27 & 25.25 & 23.25 & 21.70 \\
\hline & NLFMT [35] & 29.52 & 23.55 & 20.08 & 17.66 & 15.84 \\
\hline & GBFMT [34] & 29.56 & 23.58 & 20.10 & 17.69 & 15.87 \\
\hline & RBF [36] & 30.20 & 28.59 & 28.54 & 24.03 & 21.63 \\
\hline & MRF [37] & 30.64 & 26.61 & 20.80 & 17.99 & 16.32 \\
\hline & SBF [38] & 32.18 & 25.78 & 20.79 & 17.44 & 15.13 \\
\hline & Proposed & 33.64 & 29.06 & 26.33 & 24.13 & 22.48 \\
\hline \multirow{10}{*}{ House } & NLGRTV [30] & 26.87 & 26.62 & 25.48 & 25.24 & 25.67 \\
\hline & Ker. Reg. [31] & 29.94 & 30.89 & 30.57 & 24.44 & 17.52 \\
\hline & TV1[32] & 31.78 & 29.93 & 28.49 & 27.19 & 25.47 \\
\hline & Bitonic [33] & 31.89 & 29.59 & 27.29 & 25.51 & 24.00 \\
\hline & NLFMT [35] & 28.13 & 22.12 & 18.67 & 16.31 & 14.58 \\
\hline & GBFMT [34] & 28.14 & 22.14 & 18.68 & 16.33 & 14.60 \\
\hline & RBF [36] & 31.75 & 30.98 & 29.69 & 27.19 & 25.37 \\
\hline & MRF [37] & 32.80 & 26.39 & 19.15 & 16.51 & 14.93 \\
\hline & SBF [38] & 33.23 & 26.11 & 20.95 & 17.60 & 15.14 \\
\hline & Proposed & 35.03 & 31.33 & 28.98 & 27.21 & 25.75 \\
\hline \multirow{10}{*}{ PAN } & NLGRTV [30] & 17.43 & 17.26 & 19.00 & 16.65 & 16.73 \\
\hline & Ker. Reg. [31] & 23.90 & 23.16 & 20.50 & 18.61 & 15.76 \\
\hline & TV1[32] & 19.45 & 18.94 & 18.39 & 17.85 & 17.41 \\
\hline & Bitonic [33] & 20.16 & 19.88 & 19.48 & 16.91 & 14.74 \\
\hline & NLFMT [35] & 28.33 & 22.41 & 19.02 & 16.68 & 14.94 \\
\hline & GBFMT [34] & 28.37 & 22.45 & 19.07 & 16.72 & 14.97 \\
\hline & RBF [36] & 19.54 & 19.37 & 19.14 & 18.84 & 18.40 \\
\hline & MRF [37] & 26.88 & 23.27 & 19.24 & 16.77 & 15.18 \\
\hline & SBF [38] & 28.62 & 23.44 & 19.60 & 16.91 & 14.74 \\
\hline & Proposed & 28.72 & 23.58 & 20.79 & 19.66 & 18.56 \\
\hline
\end{tabular}

image, however our PSNR performance is best when the noise level rises from low to high levels. Altogether, PSNR performance varies dramatically with rising noise levels for all denoising methods except ours. As a result, our algorithm is capable to provide stable and consistent performance across the wide range of noise levels. As we progress from standard deviation 10 to 50, the PSNR rates for methods other than our proposed method drop dramatically. For example, at $\sigma$ (standard deviation) 10 and 50, the PSNR value for House and MRI image in SBF decreases from 33.23 to 15.14 and 32.18 to 15.13 , respectively, resulting in a difference of 18.09 and 17.05. For image House, MRI and PAN, the difference between PSNR value on $\sigma$ (standard deviation) 10 and 50 for our algorithm is less than other methods. In case of House, MRI and PAN image, however the difference between PANR value on $\sigma$ (standard deviation) 10 and 50 for NLGRTV and TV1 had less differences, but their total PSNR performance is significantly low when compared to the other approaches. Bitonic and RBF had the least differences in PSNR values for image House, MRI and PAN. However, on standard deviation of 10 to 50 , the overall PSNR value for House, MRI, and PAN image with our method is more than other comparative approaches. Therefore, the key benefits of our algorithm are being able to exhibits consistent performance with low noise contents. Our proposed technology has a far lower retardation rate than current denoising strategies. As a result, our algorithm may be used to deal with a wide range of noise levels. Execution time of proposed algorithm was found to be 4.25 seconds. Stage 1 and 2 denoising process take 2.45 seconds and 2.00 seconds, respectively. This can be further lowered by using specialised hardware with the high configuration. Also, our proposed method exhibits faster experimental operation than other comparative algorithms and can be used for effective denoising process for numerous image datasets.

\section{CONCLUSION}

In this paper, we introduce an effective denoising algorithm by the decomposition of the image in coarser and finer layers and their subsequent processing. The proposed method maintains edges in the images in case of low to high noise levels. In this method, the advantages of both spatial and transform domain techniques are employed to handle the challenges of increasing noise levels in various image datasets. In comparison to SBF, MRF, RBF, NLFMT, GBFMT, and bitonic filter, the proposed method exhibits high stability and consistency for different 
noise levels. The proposed method shows comparable performance in case of low noise level; however, it has shown great potential to perform much better performance at moderate to high noise levels. The proposed algorithm is capable to reduce noise while simultaneously preserving the edges in the images. When there is an unknown quantity of noise in different type of image datasets, the proposed method can be used as single denoising solution.

\section{Conflict of interest}

The author declares that he has no conflicts of interest.

\section{Ethical approval}

This article does not contain any studies with human participants or animals performed by any of the authors.

\section{Research involving human participants and/or animals}

This article does not contain any studies with human participants performed by any of the authors.

\section{Informed consent}

Informed consent was obtained from all individual participants included in the study.

\section{REFERENCES}

[1] Nam, Seonghyeon, Youngbae Hwang, Yasuyuki Matsushita, and Seon Joo Kim. "A holistic approach to crosschannel image noise modeling and its application to image denoising." In Proceedings of the IEEE conference on computer vision and pattern recognition, pp. 1683-1691. 2016.

[2] Mildenhall, Ben, Jonathan T. Barron, Jiawen Chen, Dillon Sharlet, Ren Ng, and Robert Carroll. "Burst denoising with kernel prediction networks." In Proceedings of the IEEE Conference on Computer Vision and Pattern Recognition, pp. 2502-2510. 2018.

[3] Foi, Alessandro, Mejdi Trimeche, Vladimir Katkovnik, and Karen Egiazarian. "Practical Poissonian-Gaussian noise modeling and fitting for single-image raw-data." IEEE Transactions on Image Processing 17, no. 10 (2008): 1737-1754.

[4] Liang, Zhetong, Jun Xu, David Zhang, Zisheng Cao, and Lei Zhang. "A hybrid 11-10 layer decomposition model for tone mapping." In Proceedings of the IEEE conference on computer vision and pattern recognition, pp. 4758-4766. 2018.

[5] Xu, Jun, Hui Li, Zhetong Liang, David Zhang, and Lei Zhang. "Real-world noisy image denoising: A new benchmark." arXiv preprint arXiv:1804.02603 (2018).

[6] Lefkimmiatis, Stamatios. "Universal denoising networks: a novel CNN architecture for image denoising." In Proceedings of the IEEE conference on computer vision and pattern recognition, pp. 3204-3213. 2018.

[7] Elad, M., \& Aharon, M. (2006). Image denoising via sparse and redundant representations over learned dictionaries. IEEE Transactions on Image processing, 15(12), 3736-3745.

[8] Ulyanov, Dmitry, Andrea Vedaldi, and Victor Lempitsky. "Deep image prior." In Proceedings of the IEEE conference on computer vision and pattern recognition, pp. 9446-9454. 2018.

[9] Ren, Dongwei, Wangmeng Zuo, Qinghua Hu, Pengfei Zhu, and Deyu Meng. "Progressive image deraining networks: A better and simpler baseline." In Proceedings of the IEEE/CVF Conference on Computer Vision and Pattern Recognition, pp. 3937-3946. 2019.

[10] Buades, Antoni, Bartomeu Coll, and J-M. Morel. "A non-local algorithm for image denoising." In 2005 IEEE Computer Society Conference on Computer Vision and Pattern Recognition (CVPR'05), vol. 2, pp. 60-65. IEEE, 2005.

[11] Dong, Weisheng, Guangming Shi, and Xin Li. "Nonlocal image restoration with bilateral variance estimation: a low-rank approach." IEEE transactions on image processing 22, no. 2 (2012): 700-711.

[12] Pajot, Arthur, Emmanuel de Bezenac, and Patrick Gallinari. "Unsupervised adversarial image reconstruction." In International Conference on Learning Representations. 2018.

[13] Mao, Xiaojiao, Chunhua Shen, and Yu-Bin Yang. "Image restoration using very deep convolutional encoderdecoder networks with symmetric skip connections." Advances in neural information processing systems 29 (2016): 2802-2810. 
[14] Dabov, Kostadin, Alessandro Foi, Vladimir Katkovnik, and Karen Egiazarian. "Color image denoising via sparse 3D collaborative filtering with grouping constraint in luminance-chrominance space." In 2007 IEEE International Conference on Image Processing, vol. 1, pp. I-313. IEEE, 2007.

[15] Gu, Shuhang, Qi Xie, Deyu Meng, Wangmeng Zuo, Xiangchu Feng, and Lei Zhang. "Weighted nuclear norm minimization and its applications to low level vision." International journal of computer vision 121, no. 2 (2017): 183-208.

[16] Plötz, Tobias, and Stefan Roth. "Neural nearest neighbors networks." arXiv preprint arXiv:1810.12575 (2018).

[17] Dabov, Kostadin, Alessandro Foi, Vladimir Katkovnik, and Karen Egiazarian. "BM3D image denoising with shape-adaptive principal component analysis." In SPARS'09-Signal Processing with Adaptive Sparse Structured Representations. 2009.

[18] Hou, Yingkun, Chunxia Zhao, Deyun Yang, and Yong Cheng. "Comments on" image denoising by sparse 3d transform-domain collaborative filtering." IEEE Transactions on Image Processing 20, no. 1 (2010): 268-270.

[19] Zontak, Maria, Inbar Mosseri, and Michal Irani. "Separating signal from noise using patch recurrence across scales." In Proceedings of the IEEE Conference on Computer Vision and Pattern Recognition, pp. 1195-1202. 2013.

[20] Buades, A., Coll, B., \& Morel, J. M. (2005, June). A non-local algorithm for image denoising. In 2005 IEEE Computer Society Conference on Computer Vision and Pattern Recognition (CVPR'05) (Vol. 2, pp. 60-65). IEEE. [21] Dabov, Kostadin, Alessandro Foi, Vladimir Katkovnik, and Karen Egiazarian. "Image denoising by sparse 3-D transform-domain collaborative filtering." IEEE Transactions on image processing 16, no. 8 (2007): 20802095.

[22] Foi, Alessandro, Vladimir Katkovnik, and Karen Egiazarian. "Pointwise shape-adaptive DCT for highquality denoising and deblocking of grayscale and color images." IEEE transactions on image processing 16, no. 5 (2007): 1395-1411.

[23] Elad, Michael, and Dmitry Datsenko. "Example-based regularization deployed to super-resolution reconstruction of a single image." The Computer Journal 52, no. 1 (2009): 15-30.

[24] Zoran, Daniel, and Yair Weiss. "From learning models of natural image patches to whole image restoration." In 2011 International Conference on Computer Vision, pp. 479-486. IEEE, 2011.

[25] Chen, Fei, Lei Zhang, and Huimin Yu. "External patch prior guided internal clustering for image denoising." In Proceedings of the IEEE international conference on computer vision, pp. 603-611. 2015.

[26] Guo, Kanghui, and Demetrio Labate. "Optimally sparse multidimensional representation using shearlets." SIAM journal on mathematical analysis 39, no. 1 (2007): 298-318.

[27] Easley, Glenn R., Demetrio Labate, and Flavia Colonna. "Shearlet-based total variation diffusion for denoising." IEEE Transactions on Image processing 18, no. 2 (2008): 260-268.

[28] Yang, Hong-Ying, Xiang-Yang Wang, Pan-Pan Niu, and Yang-Cheng Liu. "Image denoising using nonsubsampled shearlet transform and twin support vector machines." Neural networks 57 (2014): 152-165.

[29] Guorong, Gao, Xu Luping, and Feng Dongzhu. "Multi-focus image fusion based on non-subsampled shearlet transform." IET Image Processing 7, no. 6 (2013): 633-639.

[30] Liu, Qiegen, Biao Xiong, and Minghui Zhang. "Adaptive sparse norm and nonlocal total variation methods for image smoothing." Mathematical problems in engineering 2014 (2014).

[31] Takeda, Hiroyuki, Sina Farsiu, and Peyman Milanfar. "Deblurring using regularized locally adaptive kernel regression." IEEE transactions on image processing 17, no. 4 (2008): 550-563.

[32] Chambolle, Antonin, Vicent Caselles, Daniel Cremers, Matteo Novaga, and Thomas Pock. "An introduction to total variation for image analysis." In Theoretical foundations and numerical methods for sparse recovery, pp. 263-340. de Gruyter, 2010.

[33] Treece, Graham. "The bitonic filter: linear filtering in an edge-preserving morphological framework." IEEE Transactions on Image Processing 25, no. 11 (2016): 5199-5211.

[34] Kumar, BK Shreyamsha. "Image denoising based on gaussian/bilateral filter and its method noise thresholding." Signal, Image and Video Processing 7, no. 6 (2013): 1159-1172.

[35] Kumar, BK Shreyamsha. "Image denoising based on non-local means filter and its method noise thresholding." Signal, image and video processing 7, no. 6 (2013): 1211-1227.

[36] Chaudhury, Kunal N., and Kollipara Rithwik. "Image denoising using optimally weighted bilateral filters: A sure and fast approach." In 2015 IEEE International Conference on Image Processing (ICIP), pp. 108-112. IEEE, 2015 .

[37] Rangarajan, Anand, and R. Chellappa. "Markov random eld models in image processing,"." The Handbook of Brain Theory and Neural Networks, MA Arbib, ed (1995): 564-567.

[38] Tomasi, Carlo, and Roberto Manduchi. "Bilateral filtering for gray and color images." In Sixth international conference on computer vision (IEEE Cat. No. 98CH36271), pp. 839-846. IEEE, 1998.

[39] Goyal, Bhawna, Ayush Dogra, Sunil Agrawal, Balwinder Singh Sohi, and Apoorav Sharma. "Image denoising review: From classical to state-of-the-art approaches." Information fusion 55 (2020): 220-244. 
[40] Goyal, Bhawna, Ayush Dogra, Sunil Agrawal, and Balwinder Singh Sohi. "Two-dimensional gray scale image denoising via morphological operations in NSST domain \& bitonic filtering." Future Generation Computer Systems 82 (2018): 158-175.

[41] Goyal, Bhawna, Ayush Dogra, Sunil Agrawal, and B. S. Sohi. "A three stage integrated denoising approach for grey scale images." Journal of Ambient Intelligence and Humanized Computing (2018): 1-16.

[42] Liu, Yanfang, Shiqiang Li, and Heng Zhang. "Multibaseline Interferometric Phase Denoising Based on Kurtosis in the NSST Domain." Sensors 20, no. 2 (2020): 551.

[43] Routray, Sidheswar, Prince Priya Malla, Sunil Kumar Sharma, Sampad Kumar Panda, and G. Palai. "A new image denoising framework using bilateral filtering based non-subsampled shearlet transform." Optik 216 (2020): 164903.

[44] Diwakar, Manoj, and Prabhishek Singh. "CT image denoising using multivariate model and its method noise thresholding in non-subsampled shearlet domain." Biomedical Signal Processing and Control 57 (2020): 101754. [45] Sharma, Abhishek, and Vijayshri Chaurasia. "MRI denoising using advanced NLM filtering with nonsubsampled shearlet transform." Signal, Image and Video Processing (2021): 1-9.

[46] Chakraborty, Alakananda, Muskan Jindal, Eshan Bajal, Prabhishek Singh, Manoj Diwakar, Chandrakala Arya, and Amrendra Tripathi. "A multi-level method noise based image denoising using convolution neural network." In Journal of Physics: Conference Series, vol. 1854, no. 1, p. 012040. IOP Publishing, 2021.

[47] Quan, Yuhui, Yixin Chen, Yizhen Shao, Huan Teng, Yong Xu, and Hui Ji. "Image denoising using complexvalued deep CNN." Pattern Recognition 111 (2021): 107639.

[48] Ren, Chao, Xiaohai He, Chuncheng Wang, and Zhibo Zhao. "Adaptive Consistency Prior Based Deep Network for Image Denoising." In Proceedings of the IEEE/CVF Conference on Computer Vision and Pattern Recognition, pp. 8596-8606. 2021.

[49] Qiu, Chenhui, Yuanyuan Wang, Huan Zhang, and Shunren Xia. "Image fusion of CT and MR with sparse representation in NSST domain." Computational and mathematical methods in medicine 2017 (2017).

[50] Du, Jiao, Weisheng Li, Ke Lu, and Bin Xiao. "An overview of multi-modal medical image fusion." Neurocomputing 215 (2016): 3-20.

[51] Easley, Glenn, Demetrio Labate, and Wang-Q. Lim. "Sparse directional image representations using the discrete shearlet transform." Applied and Computational Harmonic Analysis 25, no. 1 (2008): 25-46.

[52] Rousselle, Fabrice, Claude Knaus, and Matthias Zwicker. "Adaptive rendering with non-local means filtering." ACM Transactions on Graphics (TOG) 31, no. 6 (2012): 1-11.

[53] Tomasi, Carlo, and Roberto Manduchi. "Bilateral filtering for gray and color images." In Sixth international conference on computer vision (IEEE Cat. No. 98CH36271), pp. 839-846. IEEE, 1998.

[54] Buades, Antoni, Bartomeu Coll, and Jean-Michel Morel. "A review of image denoising algorithms, with a new one." Multiscale modeling \& simulation 4, no. 2 (2005): 490-530.

[55] https://www.mathworks.com/matlabcentral/fileexchange/67703-image-processing-dataset-for-color-greyimage-fusion--image-blending--image-denoising--enhancement [Accessed on 15.06.2016] 